4

\title{
Threonine phosphorylation regulates the molecular assembly and signaling of EGFR in cooperation with membrane lipids
}

\author{
Ryo Maeda ${ }^{1}$, Hiroko Tamagaki-Asahina ${ }^{2}$, Takeshi Sato ${ }^{2}$, Masataka Yanagawa ${ }^{1}$, and \\ Yasushi Sako ${ }^{1,3, *}$
}

${ }^{1}$ Cellular Informatics Laboratory, RIKEN CPR, Wako, Saitama 351-0198, Japan; ${ }^{2}$ Kyoto

Pharmaceutical University, 5, Misasagi-cho, Yamashina, Kyoto, 607-8414, Japan;

${ }^{3}$ CREST JST, 4-1-8, Honcho, Kawaguchi, 332-0012, Japan

*To whom correspondence should be addressed. E-mail: sako@ riken.jp 


\section{Abstract}

16 The cytoplasmic domain of the receptor tyrosine kinases (RTKs) plays roles as a 17 phosphorylation enzyme and a protein scaffold but the regulation of these two functions 18 is not fully understood. We here analyzed assembly of the transmembrane (TM)19 juxtamembrane (JM) region of EGFR, one of the best studied species of RTKs, by 20 combining single-pair FRET imaging and a nanodisc technique. The JM domain of EGFR 21 contains a threonine residue that is phosphorylated after ligand association. We observed 22 that the TM-JM peptides of EGFR form anionic lipid-induced dimers and cholesterol23 induced oligomers. The two forms involve distinct molecular interactions, with a bias 24 towards oligomer formation upon threonine phosphorylation. We further analyzed the 25 functions of whole EGFR molecules, with or without a threonine to alanine substitution 26 in the JM domain, in living cells. The results suggested an autoregulatory mechanism in which threonine phosphorylation of the JM domain causes a switch from kinase activation dimers to scaffolding oligomers. 


\section{Introduction}

Epidermal growth factor receptor (EGFR) is an RTK responsible for cell proliferation and differentiation $(1,2)$ and consists of five domains; an extracellular domain that interacts with extracellular ligands, a single-pass transmembrane (TM) helix, a juxtamembrane (JM) domain, a cytoplasmic kinase domain, and a C-terminal tail domain for interaction with various cytoplasmic proteins $(3,4)$. Ligand association changes the conformation of EGFR in its extracellular domain (5) and induces formation of an asymmetric dimer of the intracellular kinase domains (6). This dimerization subsequently results in the phosphorylation of tyrosine residues on the tail domain and the recruitment of intracellular signal proteins such as GRB2 and PLC $\gamma$ containing SH2 and/or PTB domains (7). Although the atomic structures of most of the EGFR domains excluding the tail domain have been elucidated individually $(5,6,8-10)$, the overall architecture of this protein has not yet been revealed, leaving several unanswered questions about the molecular mechanisms underlying its functions. The correlation between the arrangement of EGFR molecules and their function is therefore still controversial, e.g., it has long been established that the dimerization of EGFR is necessary and sufficient for kinase activation (11), whereas several studies have reported the importance of higher-order oligomerization for EGFR-mediated signal transduction (12-14).

The TM helix and the JM domain (TM-JM) of EGFR play important roles in the conformational coupling of ligand binding to its activation and oligomerization $(9,11$, 15). Previous NMR studies and molecular dynamics simulations have suggested that the TM domain forms an $\alpha$-helix dimer that undergoes a change in the interaction between GXXXG motives $(9,16,17)$ following the ligand association with its extracellular domains $(17,18)$. This information regarding conformational changes in the TM dimer is then transmitted to the JM domain which comprises a JM-A (N-terminal half) region that can form an antiparallel helix dimer, and a JM-B (C-terminal half) region which makes intramolecular contact with the kinase domain (11). Both these JM regions contribute to the stable formation of an asymmetric kinase dimer conformation, which is crucial for kinase activation. The JM-A domain is rich in Lys and Arg residues, several of which are thought to interact with anionic lipid molecules of the plasma membrane and promote antiparallel dimer formation (19-21). In addition to the phospholipid species, cholesterol is a major component of the plasma membrane, mainly distributed as lipid rafts and caveolae, and has been implicated in the regulation of membrane fluidity and receptor function. Previous studies have shown that EGFR molecules are clustered in lipid rafts $(14,22)$, suggesting an interaction with cholesterols. Of note in this regard, it has been reported that the depletion of cholesterols induces various effects on EGFR signaling, 
66 also this remains controversial (23-25). Another important factor in the regulation of 67 EGFR through the TM-JM is the phosphorylation of Thr654 at the JM-A domain. 68 Although Thr phosphorylation is known to be involved in EGFR deactivation, the precise 69 mechanism of this is still elusive (26).

In our present study, by combining single-pair FRET measurements and nanodisc technology, we studied how the functions of anionic lipids, cholesterols, and EGFR Thr654 phosphorylation (pT654) are orchestrated to achieve the regulation of dimerization and/or oligomerization of EGFR. We previously reported that anionic lipids cause the dimerization of JM domains, and that pT654 together with acidic lipids induces the dissociation of the EGFR dimer (20). In this current study, we report that both the TM and JM protomers of EGFR are positioned closer to each other in the presence of cholesterols than in the EGFR dimers promoted by anionic lipids. Furthermore, we found that TM-JM peptides were oligomerized in cholesterol containing membranes, which was promoted by pT654. Finally, in living cells expressing whole EGFR molecules, we observed differential functional roles of this crucial signaling factor that are dependent on the pT654 levels. Our results suggest that the membrane cholesterol and pT654 cooperatively shift the EGFR function from the kinase dimer to the scaffold oligomer. 


\section{Results}

\section{Incorporation of TM-JM peptides into nanodiscs}

Synthesized peptides of the TM-JM region of EGFR were prepared and labeled with a fluorophore $\mathrm{Cy} 3$ or $\mathrm{Cy} 5$ at the $\mathrm{N}$-terminus (TM terminal region) or C-terminus (JM terminal region), respectively (Fig. 1a). The peptides were reconstituted into nanodisc structures with membrane scaffold proteins (MSPs) and lipid molecules (Fig. 1b, c). Mixtures of POPC (PC), POPS (PS), and cholesterol were used for reconstruction (Fig. 1d). The nanodiscs containing cholesterol showed two peaks following size exclusion gel chromatography, one of which had a smaller disc size relative to that without cholesterol (Fig. 1e). To avoid the effects of disc size, we collected and used nanodiscs involved in the first peak fraction which had a similar size without cholesterol. This fraction was composed of the same ratio of lipids as used in the preparation (Fig. 1f). Synthesized TMJM peptides with pT654 were also reconstituted into nanodiscs. The nanodisc construction was examined under a transmission electron microscope (Fig. 1g). In total, 16 types of nanodiscs were applied to subsequent single-molecule measurements. Fractions of PS (inner leaflet) and cholesterol (inner and outer leaflets) mimicked those in the plasma membrane.

\section{TM-TM interaction in the EGFR dimer}

Nanodiscs containing Cy3 and Cy5-labeled peptides were immobilized onto glass surfaces and illuminated with a 532-nm laser for $\mathrm{Cy} 3$ excitation. A portion of the fluorescent spots contained Cy5 fluorescence derived from the occurrence of FRET (Fig. $2 \mathrm{a}, \mathrm{b})$. Based on the fluorescence intensity, we selected nanodiscs containing one Cy3and one Cy5-labeled peptide and calculated the FRET efficiency, $E_{\mathrm{FRET}}$ (Fig. 2c).

We first examined the interactions between the $\mathrm{N}$-terminal regions of the TM domains (Fig. 3). When Thr654 was not phosphorylated and the membrane contained only PC as lipid species, $E_{F R E T}$ distributed with a peak at a relatively high ( $>0.90$; Table I) value (Fig. 3a), indicating close proximity of the two TM domains. There may be additional stable structures between the TM domains, as suggested by the small peaks and shoulders in the $E_{F R E T}$ distribution. The addition of anionic lipid PS caused few effects, i.e., the TM dimers were maintained as the major structure (Fig. 3b). Peptides with pT654 slightly decreased the major peak positions of the $E_{F R E T}$ distributions in the PC or PC/PS membranes (Fig. 3e, f). The smooth distribution of the pT654 peptides suggested that pThr654 had homogenized possible substructures of the TM dimers of nonphosphorylated peptides. PS had a little effect on the TM-TM interactions regardless of the Thr654 phosphorylation. The presence of cholesterol in the membrane concentrated 
120 the distributions to a high $E_{F R E T}(>0.95)$ region (Fig. 3c, g, h), indicating that the N121 terminal regions of TM-TM dimers were positioned in extremely close proximity. 122 Although the peak position was similar in PC and PC/cholesterol membranes (Fig 3a, c;

123 Table I), the distribution in PC/cholesterol membrane was more accumulated at the peak position. It also should be noted that the accumulation at a high $E_{F R E T}$ region was a remarkable observation for pT654 peptides. Thus, pT654 and the presence of membrane cholesterol decreased the distance between the two N-termini of the TM domains in cooperation. PS competed with cholesterol when Thr654 was non-phosphorylated (Fig. $3 d)$.

\section{JM-JM interaction in the EGFR dimer}

131 To examine the effects of lipid species and pT654 on JM-JM interaction, $E_{F R E T}$ 132 distributions were determined under the C-terminus labeling (Fig. 4). In PC membrane, $133 E_{F R E T}$ was broadly distributed with a peak around 0.81 (Fig. 4a; Table I). It is plausible that the JM-A dimers are fluctuating between minor dissociation and major association states. In the PC/PS membrane, the peak fraction was increased, indicating that PS stabilized the JM-A dimer conformation (Fig. 4b). pThr654 increased the low FRET fraction in the PC/PS membrane (Fig. 4f) but showed little effect in the PC membrane (Fig. 4e). These results confirmed the results of our previous study (20). Cholesterol moved the $E_{F R E T}$ peak between non-phosphorylated peptides to higher values (0.93-0.94) regardless of whether it was a PC or PC/PS membrane (Fig. 4c, d), i.e., cholesterol forced the C-termini of the JM-A domains to position closer.

The mixed effects of cholesterol and pT654 on the JM-JM interaction were further examined. In the PC membrane (Fig. 4g), cholesterol increased the high FRET

144 population to a comparable level to those shown for non-phosphorylated peptides.

145 Cholesterol in the PC/PS membrane (Fig. 4h) reversed the $E_{F R E T}$ distribution seen in the 146 PC/PS membrane without cholesterol (Fig. 4f) to that observed in the PC membrane (Fig. 4e) i.e. minor low FRET and major high FRET states in the PC/PS/cholesterol membrane. However, it should be noted that the $E_{F R E T}$ values were not as large as those found in other conditions with cholesterol (Fig. 4c, d, g), i.e., the cholesterol effect on JM-JM interaction was partially diminished by the coexistence of PS and pThr654. Overall, our data showed that cholesterol increased the proximity between the C-terminus of JM domains in PC and PC/PS membranes, and that this effect overrode that of pThr654 in the PC/PS membrane. 
156 We speculated that the accumulation of EGFR in lipid rafts, which has been reported in previous studies, could be an effect of cholesterol in the raft membrane. We examined the assembly of TM-JM peptides in the nanodiscs, collecting images of fluorescent spots containing only Cy3-labeled peptides to avoid interference from the effects of FRET occurring between $\mathrm{Cy} 3$ and Cy5. Figure 5 displays the fluorescence intensity histograms of C-terminus-labeled TM-JM peptides in nanodiscs containing or not-containing cholesterol. Cholesterol shifted the histograms toward higher intensities for the pT654 peptides (Fig. 5b, d), suggesting a cooperative effect of cholesterol and Thr phosphorylation to induce higher-order assembly of the TM-JM peptides.

\section{Assembly of TM regions}

167 For analysis of the interactions between more than two TM or JM domains in the 168 assembled structures at the N-terminus, images of fluorescent spots containing two Cy3169 labeld peptides and one Cy5-labeled peptide were collected based on their 2-color 170 fluorescence trajectories (Fig. 6a). These nanodiscs showed a variety of Cy3 donor 171 fluorescence intensities before Cy5 photobleaching indicating that the three peptides 172 interacted diversely. We constructed maximum fluorescence intensity histograms in the 173 Cy3 donor channel before and after Cy5 acceptor photobleaching for inference of the 174 interactions between three TM domains (Fig. 6b-i). In all conditions other than non175 phosphorylated peptides in the PC/PS membrane, Cy3 distributions after Cy5 176 photobleaching (red) peaked at the fluorescence intensity of $\sim 100$ (in arbitrary units), 177 which was smaller than that observed for the C-terminal-labeled peptides $(\sim 150$; Fig. $7 \mathrm{~b}-$ 178 i). This result must have been caused by homo-FRET (self-quenching) between two N179 terminal labeled $\mathrm{Cy} 3$ peptides. Together with the very small intensity peaks prior to Cy5 180 photobleaching (blue), these distributions suggested that TM regions of the three peptides 181 (two of them were randomly labeled with Cy3) were oligomerized in very close proximity 182 to each other in the major configuration (trimer; Fig. 6j).

183 For non-phosphorylated peptides in the PC/PS membrane however (Fig. 6c), the 184 Cy3 intensity histogram after Cy5 photobleaching (red) had a peak intensity at $\sim 150$, 185 indicating that two Cy3-labeled peptides in the major population were positioned 186 separately. In addition, the low intensity shoulder in this distribution indicated the presence of proximate dimers (and trimers). Taken together, these distributions suggested that N-terminal regions of three non-phosphorylated peptides have a stronger tendency to 189 arrange as one dimer and one monomer in the PC/PS membrane than any other condition. 190 A similar dimer + monomer arrangement might be contained in the distributions under other conditions as a minor fraction. Consistent with this suggestion, for the non- 
192 phosphorylated peptides in PC/PS membrane before Cy5 photobleaching (Fig. 6c, blue),

193 a homo-FRET fraction ( 100; with a low $E_{F R E T}$ to Cy5) was evident compared to other 194 conditions. It should be noted that the ability of PS to promote the dimer + monomer 195 arrangement was diminished for pT654 peptides (Fig. 6g). Cholesterol also reduced this 196 effect of PS even for non-phosphorylated peptides (Fig. 6e). As observed in the earlier analysis of the TM-JM peptide dimer (Fig. 3), the effects of PS and cholesterol were competitive.

\section{Assembly of JM regions}

201

We constructed Cy3 fluorescence intensity histograms of two Cy3 and one Cy5 peptide with C-terminal-labeling in single nanodiscs in order to analyze the interactions between three JM domains (Fig. 7). The distributions of the Cy3 florescence after Cy5 photobleaching (red) were similar under all conditions, exhibiting a single peak at $\sim 150$, which was the fluorescence intensity of the two $\mathrm{Cy} 3$ molecules without strong interactions to induce homo-FRET. Both the trimer and dimer + monomer arrangements are possible if we assume that the three molecules in the trimer and two molecules in the dimer are not so close that they will induce homo FRET (Fig. 7j).

Prior to Cy5 photobleaching (blue), the distribution peaks were observed in the region of small $\mathrm{Cy} 3$ intensities indicating the proximity of both $\mathrm{Cy} 3$ molecules with $\mathrm{Cy} 5$ to induce high $E_{F R E T}$, as observed between two molecules in a nanodisc (Fig. 4), i.e., the formation of a JM trimer. An accumulation in the low intensity peak fraction was very evident for non-phosphorylated peptides in the membranes containing cholesterol (Fig. $7 \mathrm{~d}, \mathrm{e})$. On the other hand, fractions at the intensities similar to those observed after Cy5 photobleaching were significant for pT654 peptides in the membrane without cholesterol (Fig. 7f, g). In general, pT654 peptides exhibited higher fluorescence intensity compared to non-phosphorylated peptides in the corresponding membrane lipid compositions. One possible explanation is that the fraction of high $\mathrm{Cy} 3$ intensity before $\mathrm{Cy} 5$ photobleaching represents a $\mathrm{Cy} 3$ dimer in the dimer + monomer arrangement of three peptides (Fig. 7j). Another possibility is that it was caused by an increased distance between three JM domains in trimers, resulting from Thr phosphorylation to reduce $E_{F R E T}$ (Fig. $7 \mathrm{j}$ top middle). These two arrangements could potentially coexist.

Considering the possible arrangement for the TM and JM regions of three TMJM peptides together (Figs. 6 and 7), we conclude that cholesterol induces the closely proximate oligomerization of three JM domains of non-phosphorylated peptides whereas PS preferentially causes a dimer + monomer arrangement, and the Thr phosphorylation 
228 domains in the presence of cholesterols (Fig. 8). Because of the limitation of FRET measurement, we could not distinguish whether the peptides in the oligomers directly interacted or not.

\section{Effect of Thr phosphorylation on the Tyr phosphorylation of EGFR}

233 Our single-molecule structural analysis suggested that pT654 is a key regulator of the molecular assembly of EGFR, which may affect its functions. We examined this possibility in living cells. It is known that PKC activation under EGF signaling induces pT654 in EGFR. This process has been thought to be a negative feedback pathway in the EGFR system. We expressed a wild type (wt) or T654A mutant EGFR in CHO-K1 cells, which have no intrinsic expression of EGFR. An increase in pT654 was observed for wt EGFR after EGF stimulation, and treatment of these cells with phorbol-12-myristate 13acetate (PMA), a PKC activator, caused stronger phosphorylation of Thr654 regardless of EGF stimulation (Fig. 9a). Application of a saturation amount (100 ng/ml) of EGF to the culture medium induced phosphorylation of Tyr1068 (pY1068) of both the wt and T654A mutant EGFR proteins (Fig. 9b). pY1068 is a major association site on EGFR for the adaptor protein GRB2 and its levels after EGF stimulation were significantly increased by the T654A mutation compared to wt, as expected from the negative effect of pT654. Whereas, pretreatment with PMA decreased the pY1068 level in both the wt and T654A mutant EGFR (Fig. 9c). The PMA-induced decrease in pY1068 for the wt protein could be a negative effect of increased pT654, but the similar result with the T654A mutant suggests that PMA has indirect effects independent of pT654.

\section{Single-molecule imaging of the clustering and movement of EGFR}

We expressed EGFR (wt and T654A) fused with GFP in CHO-K1 cells and, by using single-molecule imaging, detected cluster size distributions and lateral diffusion movements of EGFR molecules in the plasma membrane (Fig. 10a). Clustering of EGFR was measured as the fluorescence intensity distribution of EGFR spots, and the lateral diffusion movements were measured as the increase in the mean square displacement (MSD) of the spots with time. Both measurements were performed before and after 10 min of EGF application to the medium. Application of EGF to the medium induced clustering and immobilization of wt EGFR as we have reported previously(14, 27). The distributions of EGFR cluster size suggest formation of oligomers containing up to more than 10 molecules (Fig. 10b). The convex shapes of MSD curve with time indicate subdiffusion of EGFR molecules (Fig. 10c).

Even in the absence of EGF, PMA treatment of cells increased fractions of 
264 higher-order wt EGFR oligomers (Fig. 10b left), though diffusion movements were hardly affected (Fig. 10c left). This oligomerization was not as strong as that induced by EGF in the absence of PMA, and application of EGF to the PMA treated cells did not induce further oligomerization at least up to $10 \mathrm{~min}$ but significantly decreased mobility. Application of EGF induced strong oligomerization and immobilized wt EGFR in cells without PMA treatment. For T654A mutant, PMA treatment hardly affected both oligomerization (Fig. 10b right) and movements (Fig. 10c right) in the absence of EGF. EGF induced strong oligomerization and immobilization of T654A mutant independent of PMA treatment. The effect of PMA to induce EGFR oligomerization dependent on Thr654 was consistent with pT654-induced oligomerization of TM-JM peptides in nanodiscs.

In summary, single-molecule measurements suggest three states of EGFR oligomerization depends on pT654 and EGF association (Fig. 10d). PMA treatment of wt EGFR but not T654A mutant induced a medial level of oligomerization, which could be stabilized by pT654. Strong oligomerization observed under the weak pT654 level in wt EGFR and no pT654 in T654A mutant was caused by a distinct mechanism of pT654. On the other hand, it is possible that immobilization of EGFR relates with its tyrosine phosphorylation levels as we have observed previously (28). Immobilization in the presence of PMA was more evident in T654A mutant in which pY1068 level was higher than that in wt EGFR (Fig. 9), and PMA treatment decreased immobilization and pY1068 in wt EGFR.

\section{Interaction of EGFR with GRB2}

We finally measured the interaction of EGFR with GRB2 in living cells using a split luciferase (NanoBiT) assay, in which the C-terminus of EGFR and the N-terminus of GRB2 were conjugated with the large BiT (LgBiT) and the small BiT (SmBiT) of NanoLuc luciferase (29), respectively. The association of EGFR and GRB2 promoted the formation of active luciferase to produce chemiluminescence emission (Fig. 11a). From the timecourses of the NanoBiT signal increases after EGF treatment of cells expressing the wt or T654A mutant EGFR (Fig. 11b), the maximum intensities indicated a dose dependent response to the EGF concentration in the medium (Fig. 11b, c). The maximum intensity was significantly increased after pretreatment with PMA in cells expressing wt EGFR, despite the fact that the pY1068 level was reduced after the PMA treatment (Fig. 9c). This effect of PMA was not observed to any extent with the T654A mutant, and the GRB2 association was not increased by this mutation in the absence of PMA, even though the pY1068 level after EGF stimulation was significantly increased in the mutant (Fig. 
300 9c). The increase in GRB2 association observed for wt EGFR after PMA treatment was

301 not likely to be an indirect effect of PMA because it was not observed for the T654A

302 mutant, in which the pY1068 level was also affected by PMA. These results suggest that

303 pT654 promotes the formation of a GRB2 recognition state for EGFR, and that the

304 inhibition of Thr654 phosphorylation prevents a GRB2-EGFR association in spite of 305 enhanced Tyr1068 phosphorylation. 


\section{Discussion}

308 We have here studied the dimerization and oligomerization of EGFR molecules by reconstituting its TM-JM peptides into nanodiscs. As expected from the positively charged JM-A sequence and accumulation of EGFR in the raft membrane, PS and cholesterol affect the molecular assembly of the TM-JM peptides. Interestingly, these two lipid species each function in a specific fashion i.e. the $E_{F R E T}$ distributions between the two TM-JM peptides in the nanodiscs suggested that PS facilitates JM dimerization, while cholesterol induces closer positioning of both the TM and JM domains (Figs. 3 and 4). In addition, cholesterol promoted the oligomeric assembly of TM-JM peptides (Figs. 6 and 7). We herein propose schematic models for the formation of the EGFR TM-JM dimers and trimers under various conditions of lipid exposure and Thr654 phosphorylation (Fig. 8), in which PS and cholesterol exert competitive effects on dimerization and oligomerization, and pT654 disrupts the PS-induced JM dimer, thus promoting oligomerization of the peptides.

It should be noted that the $E_{F R E T}$ distribution was broad in every one of our observations in this present study, especially between JM domains, indicating multiple configurations coexisting under each condition. Non-phosphorylated peptide dimers showed a peak at around $E_{F R E T}>0.90$ and $>0.81$ both for $\mathrm{N}$ - and C-terminus labeling, respectively. We can attribute this configuration to that suggested in a previous NMR study, in which two JM domains form an anti-parallel helix dimer (9). PS stabilized this configuration probably at the JM side of the non-phosphorylated peptide. Acidic lipids are known to interact with the positively charged JM-A domain $(19,30)$ whereas cholesterol induced more proximation of the two peptides at the both $\mathrm{N}$ - and C-termini in the major configuration ( $E_{F R E T} 0.95$ and 0.93 ), which must be distinct from the arrangement containing antiparallel JM helices (Fig. 8). If the TM-JM domains of whole EGFR dimer adopt similar configurations as suggested for the TM-JM peptides in the nanodiscs, the arrangement of two kinase domains indicates namely, the kinase activity would be affected by the lipid composition and by pT654 (Fig. 11d).

Cholesterol was found in our current analysis to increase the population of nanodiscs containing three TM-JM peptides with pT654 (Fig. 5). The fluorescence intensity distributions of the two $\mathrm{Cy} 3$ probes among the three peptides in the presence of cholesterol suggested that a close trimer was the major configuration (Figs. 6 and 7). The cholesterol-induced oligomerization of TM peptides with a short JM region (to T654) of EGFR in liposomes has been reported previously from NMR analysis (31). In that report however, pT654 in the peptide showed no obvious effect on the oligomerization in PC and $\mathrm{PC} /$ cholesterol liposomes (without any acidic lipids), consistent with our current and 
343 previous results indicating interplay of acidic lipids and pT654. The induction of

344 oligomerization seems to be a general effect of cholesterol upon $\alpha$-helix peptides in lipid

345 bilayers (32). Our result does not necessarily mean trimerization in the physiological

346 conditions but reflects a tendency of oligomerization. We picked up trimers in the analysis

347 to avoid complexity in the interactions between more than three molecules. While in the

348 PC/PS membrane without cholesterol (Figs. 6 and 7), the probability to adopt a one dimer

349 + one monomer configuration seems to be increased for the non-phosphorylated peptides,

350 likely because peptides have difficulty forming trimers when containing the anti-parallel

351 helix JM dimer, the pT654 event appears to dissociate the JM dimer to help in the

352 formation of the close trimer especially in the presence of cholesterol. If this assumption

353 is correct, oligomer formation will be inhibitory for EGFR kinase activity. Our observed

354 increases in the pY1068 level in the T654A mutant of EGFR (Fig. 9) support this

355 possibility. Recently, we found that cholesterol depletion induces hyperphosphorylation

356 of Y1068 and Y1173 of EGFR upon EGF stimulation. This seems to be caused by an

357 increase in the pre-dimer fraction of EGFR. However, importantly, reactions downstream

358 of EGFR autophosphorylation, including the translocation of GRB2 to the cell surface

359 and phosphorylations of SHC, AKT, and ERK were diminished under the cholesterol

360 depletion. At the same time, clustering of EGFR over dimers was strongly inhibited (33).

361 These observations are consistent with the results of this study.

362 The antiparallel helix dimer of JM is thought to facilitate asymmetric interaction

363 between the kinase domains of EGFR, and hence its activation, in order to phosphorylate

364 tyrosine residues in the C-tail $(11,34)$. This tyrosine phosphorylation results in the

365 recruitment of PKC and other threonine kinases from the cytoplasm to the EGFR

366 molecules for the phosphorylation of Thr654, which is known to negatively regulate

367 EGFR signaling $(26,35)$. Our previous results suggested that the mechanism underlying

368 this negative effect of pT654 is the dissociation of JM dimers in the presence of acidic

369 lipids (20). At the same time, pT654 might induce the oligomerization of EGFR in the

370 presence of cholesterol. Supporting this possibility, our current single-molecule imaging

371 in living cells revealed the oligomerization of unliganded wt EGFR after PMA treatment,

372 which induced pT654 (Fig. 10). In the unliganded condition, the extracellular domains of

373 EGFR are thought to prevent the JM dimerization for kinase activation $(9,36)$. Clustering

374 of EGFR is also prevented largely in the unliganded condition. However, probably due to 375 the structural fluctuation, a small part of the unliganded EGFR are oligomerized (33).

376 pT654 (PMA) stabilized the unliganded oligomers possibly destructing the JM 377 antiparallel dimers.

378 We previously reported that oligomers of EGFR formed after cell stimulation 
with EGF function as the major signal transduction sites for GRB2, where the dissociation rate constant with GRB2 was reduced increasing the affinity between single-molecules of EGFR and GRB2 (14). In addition, our current analyses found an increase in the wt EGFR/GRB2 association following PMA treatment (Fig. 11). We speculate that after the initial Tyr phosphorylation, successive Thr phosphorylation reduces Tyr kinase activity of EGFR but enhances the formation of signal transduction oligomer in the medium immobilized and oligomerized state of EGFR molecules (Fig. 10d). Further immobilization and oligomerization were found in our current experiments to be induced by EGF in the absence of PMA for wt EGFR and with or without PMA for the T654A mutant. This process might include EGFR molecules accumulated into the clathrin coated pits (37) and be independent of pT654. In the previous report (38), ERK activation was reduced by the Thr654/669 of EGFR to Ala substitution suggesting only negative effects of Thr654, however, oligomerization of EGFR was not observed in this report. Distinct from wt EGFR, the T654A mutation in EGFR suppressed the GRB2 association after EGF association in spite of the higher levels of Tyr phosphorylation at the binding site compared to wt. Thus, even though pT654 is inhibitory for EGFR kinase activity, it promotes signal transduction to the cytoplasmic protein, GRB2.

Based on our present results, we propose a model of EGFR signaling regulated by membrane lipids and Thr654 phosphorylation (Fig. 11d). The signal transduction mediated by EGFR is a complex multi-step process. Conformational changes in the extracellular domain of EGFR upon ligand association allow JM domains to form acidic lipid-facilitated anti-parallel JM helix dimers and asymmetric kinase domain dimers(11, 34). This is the activation process for EGFR kinase, in which acidic membrane lipids and cholesterol play stimulative and inhibitory roles, respectively (20, 33). Tyrosine phosphorylation in the kinase-active EGFR dimers recruits PKC from the cytoplasm (39). The association of PLC $\gamma$ to the EGFR phosphotyrosine for the degradation of $\mathrm{PIP}_{2}$ is involved in this process. PKC then phosphorylates Thr654 (40), which dissociates antiparallel JM dimers in the presence of remaining acidic lipids and supports the oligomerization of EGFR in the presence of cholesterol, especially after the removal of acidic lipids around the EGFR molecules. The cholesterol-induced oligomer of EGFR is a major site of interaction with cytoplasmic proteins including GRB2 (14). Thus, a major function of EGFR is shifted from a kinase for self-activation to a scaffold for signal transduction. Thr654 phosphorylation is a key step underlying this role change of EGFR and is not merely an inactivating mechanism. The degradation of $\mathrm{PIP}_{2}$, a major anionic lipid in the inner leaflet of the plasma membrane, may support this role change. Importantly, both Thr654 phosphorylation and $\mathrm{PIP}_{2}$ degradation are caused by the kinase 
bioRxiv preprint doi: https://doi.org/10.1101/2021.05.14.444132; this version posted September 6, 2021. The copyright holder for this preprint (which was not certified by peer review) is the author/funder, who has granted bioRxiv a license to display the preprint in perpetuity. It is made available under aCC-BY-NC-ND 4.0 International license.

415 activation of EGFR. Hence, this represents an ingenious autoregulatory process involving 416 membrane proteins and lipids.

417 


\section{Materials and Methods}

\section{Materials}

420 1-palmitoyl-2-oleoyl-sn-phosphatidylcholine

(PC), 1-palmitoyl-2-oleoyl-sn-

421 phosphatidylserine (PS), and cholesterol were purchased from Avanti Polar Lipids

422 (Alabaster, AL) as chloroform solutions (PC and PS) or powders (cholesterol). Cy3-

423 maleimide and Cy5-maleimide were purchased from GE Healthcare Life Sciences (Little

424 Chalfont, UK). n-octyl-b-D-glucoside (OG) was purchased from Dojindo (Kumamoto,

425 Japan). Monofunctional polyethylene glycol-succinimidyl valerate (s-PEG, $5000 \mathrm{~mol} w \mathrm{w}$ )

426 and biotinylated monofunctional polyethylene glycol-succinimidyl valerate (b-PEG,

$4275000 \mathrm{~mol} \mathrm{wt}$ ) were purchased from Laysan Bio (Arab, AL). Chinese hamster ovary K1

428 (CHO-K1) cells were provided from RIKEN BRC through the National Bio-Resource

429 Project (MEXT, Tokyo, Japan).

\section{Plasmid construction}

432 Construction of the cDNA of full-length human EGFR (wt) fused with GFP was described 433 previously (14). T654A mutant DNA was constructed using PrimeSTAR Max (Takara, 434 Kusatsu, Japan) in the wt EGFR vector. The primer sequences were as follows: 435 EGFR(T654A)-f: GAAGCGCGCGCTGCGGAGGCTGCTGC and EGFR(T654A)-r: 436 CCGCAGCGCGCGCTTCCGAACGATGTG, respectively. For NanoBiT assays, full437 length human EGFR (wt or T654A mutant) was fused with $\mathrm{LgBiT}$ at the C-terminus (wt 438 or T654A EGFR-LgBiT), and GRB2 was fused with SmBiT at the N-terminus (GRB2$439 \mathrm{SmBiT}$ ) as follows. The LgBiT fragment amplified from $\mathrm{pBiT1.1-C} \mathrm{[TK/LgBiT]}$ Vector 440 (Promega) using KOD One PCR Master Mix (TOYOBO) was subcloned into the AgeI441 and NotI-digested EGFP-N1 vector (Clontech), and subsequently full-length EGFR 442 fragment was subcloned into the NheI- and HindIII-digested the LgBiT-inserted EGFP443 N1 vector. The GRB2-SmBiT fragment was constructed using KOD One PCR Master 444 Mix (TOYOBO) and subcloned into the AgeI- and Sall-digested EGFP-C2 vector 445 (Clontech). The primer sequence of SmBiT was designed from pBiT2.1-N [TK/SmBiT] 446 Vector (Promega).

\section{$448 \quad$ Peptide synthesis and purification}

449 Peptides corresponding to the TM-JM regions of EGFR (618-666) were synthesized by 450 solid-phase methods with the sequence KIPSIATGMVGALLLLLVVALGIGLFM451 RRRHIVRKRT ${ }_{65}$ LRRLLQERELVE-NH $_{2}$ (28). For the experiments with the C-terminal 452 labeled EGFR peptide, peptides containing a cysteine at the $\mathrm{C}$-terminus were synthesized. 453 These synthetic peptides were purified by reverse-phase high-performance liquid 
454 chromatography on a C4 column with a gradient of 1-propanol and acetonitrile (1:1) over $455 \quad 0.1 \%$ aqueous trifluoroacetic acid. To prepare the C-terminal labeled peptide, Cy3456 maleimide or Cy5-maleimide was introduced to the sulfide group on the cysteine at the 457 C-terminus of the TM-JM peptide by mixing the peptide and the fluorescence derivative 458 in dimethyl formamide under basic conditions. For experiments with the N-terminal 459 labeled peptide, $\mathrm{Cy} 3-\mathrm{COOH}$ or $\mathrm{Cy} 5-\mathrm{COOH}$ was reacted with an elongating peptide on 460 the resin in the presence of 1-[bis(dimethylamino)methylene]-1H-benzotriazolium 3461 oxide hexafluorophosphate (HBTU) and diisopropylethylamine (DIEA), which activate 462 the carboxyl group on the fluorophore derivative. For synthesis of Thr654 phosphorylated 463 peptides, phosphorylated threonine derivatives were utilized. The purity was confirmed 464 by reverse-phase high-performance liquid chromatography and matrix-assisted laserdesorption/ionization time-of-flight mass spectroscopy analysis.

\section{Nanodisc preparation}

468 For nanodisc construction, fluorescent EGFR TM-JM peptides co-solubilized with lipids 469 and OG in hexafluoroisopropanol were first dried to form thin films. These peptide films were then resolubilized in buffer A $(0.5 \mathrm{M} \mathrm{NaCl}, 20 \mathrm{mM}$ Tris/Cl, $0.5 \mathrm{mM}$ EDTA) containing $30 \mathrm{mM} \mathrm{OG}$ and $5 \mathrm{mM}$ dithiothreitol (pH 7.5). His8-tagged MSP 1E3D1 (MSP) was expressed in $E$. coli and purified as described previously (41). The concentration of MSP was quantified based on the absorbance at $280 \mathrm{~nm}\left(29,910 \mathrm{M}^{-1} \mathrm{~cm}^{-1}\right)$. Thin PC or PS films were formed by evaporation of the solvent (chloroform) under a steam of nitrogen gas and dried in vacuum. Cholesterol powders were first dissolved in chloroform, and a thin film was formed as described above. PC, PS, and cholesterol were resuspended in buffer A containing $0.4 \mathrm{M}$ sodium cholate $(\mathrm{pH} 7.5)$ at a final concentration of $10 \mathrm{mM}$. Cy3- and Cy5-labeled TM-JM peptides in buffer A were mixed in equal amounts and then conjugated with MSP and phospholipid mixtures (PC, PC/PS, PC/cholesterol, $\mathrm{PC} / \mathrm{PS} /$ cholesterol) at a molar ratio of 1:1:120 $\mu \mathrm{M}$ (TM-JM/MSP/lipids). The mixture was dialyzed against a buffer containing $0.5 \mathrm{M} \mathrm{NaCl}, 20 \mathrm{mM}$ Tris/Cl, and $5 \mathrm{mM}$ EDTA (pH $7.5)$ at $4^{\circ} \mathrm{C}$ to reconstitute the nanodiscs by removing the detergent. The aggregates and liposomes were removed from the mixture by size-exclusion chromatography using a Superdex 200 Increase column (GE Healthcare Life Sciences) and the peak fractions containing nanodiscs of around $11 \mathrm{~nm}$ in diameter were collected.

\section{Single-pair FRET (spFRET) measurements}

488 Nanodisc samples were immobilized on the surface of a glass chamber as described previously $(20,42,43)$. Briefly, amine-modified glass surfaces were coated with $99 \%$ s- 
490 PEG and 1\% b-PEG. NeutrAvidin (Thermo Fisher Scientific、Waltham, MA) was then

491 bound to the b-PEG. The nanodisc samples bound with biotinylated anti-His8-tag 492 antibody (MBL Life Science) were loaded into the glass chamber and allowed to bind to 493 the NeutrAvidin-coated glass surface, after which unbound nanodiscs were washed away. 494 To reduce the photobleaching rate of Cy3 and Cy5, the nanodisc-loaded chamber was 495 filled with dialysis buffer containing 2-mercaptoethanol at the final concentration of $0.5 \%$ 496 (w/v). The fluorescence of Cy3 and Cy5 was observed under a TIRF microscope based 497 on an inverted microscope (Ti2; Nikon) with a 60x oil-immersion objective (ApoTIRF 498 60x 1.49 NA; Nikon). The fluorescence activity of Cy3 was excited using a $532 \mathrm{~nm}$ laser 499 (Compass 315M-100). Dual-color imaging was carried out through a 4x relay lens by 500 using two EMCCD cameras (C9100-134, ImagEM; Hamamatsu Photonics, Hamamatsu, 501 Japan) with a 200x EM gain. Images of 512 x 512 pixels $(67 \mathrm{~nm} /$ pixel $)$ were recorded 502 with a temporal resolution of $100 \mathrm{~ms}$ /frame using MetaMorph (Molecular Devices, San 503 Jose, CA) or AIS (ZIDO, Toyonaka, Japan).

\section{Analysis of FRET signals}

506 The measurement of fluorescence intensities of single nanodiscs was performed using 507 ImageJ software, as described previously (44). The background noise was filtered out 508 using the Subtract Background function in ImageJ. Fluorescence intensities of Cy3 and 509 Cy5 in single nanodiscs were measured as averages from circles with a diameter of 12 510 pixels containing a fluorescence spot. The average intensity of the same sized circles in 511 which no spot was present was subtracted as the background. Along the fluorescence 512 trajectories of TM-JM-Cy3 and TM-JM-Cy5, the FRET efficiency, $E_{\text {FRET }}$, for each frame 513 ( $>2000$ ) was calculated from the fluorescence intensities in the donor $I_{\mathrm{D}}$ and FRET $I_{\mathrm{A}}$ 514 channels as

$$
E_{F R E T}=\frac{I_{A}-\beta I_{D}}{I_{A}+(\gamma-\beta) I_{D}}
$$

where $\beta$ and $\gamma$ are coefficients for the compensation of fluorescence leakage from the donor dye to the acceptor detector channel, and the difference in the detection efficiencies of the dyes, respectively (45). Coefficients were calculated using the intensity time traces as $\beta=0.03$ and $\gamma=0.4$, respectively. $E_{F R E T}$ distributions were all different in KolmogorovSmirnov (KS) test with a rejection $\mathrm{p}<0.006$. The peak positions and their $95 \%$ percentile section were estimated using bootstrap method from a discretized $E_{F R E T}$ distribution (Table I), in which the bin width was set to 0.02 and the resampling number was 300 . KS test was done using "StatsKSTest" in Igor Pro 8.0 (WaveMetrix). Resampling for 
524 bootstrap method was done using "sample" function in R.

\section{Cell culture and transfection}

527 CHO-K1 cells were maintained in HAM F12 medium supplemented with $10 \%$ fetal 528 bovine serum at $37^{\circ} \mathrm{C}$ under $5 \% \mathrm{CO}_{2}$. HEK293S cells were maintained in DMEM F12 529 medium supplemented with $10 \%$ fatal bovine serum at $37^{\circ} \mathrm{C}$ under $5 \% \mathrm{CO} 2$. For western 530 blotting assays, DNA constructs of full-length wt and T654A EGFR $(1 \mu \mathrm{g})$ were 531 transiently transfected into CHO-K1 cells using FuGENE HD Transfection Reagent 532 (Promega, Madison, WI). For single-molecule measurements, CHO-K1 cells were 533 transfected with either a wt or T654A EGFR-GFP gene ( $0.5 \mu \mathrm{g}$ each $)$ using Lipofectamine 5343000 Reagent (Thermo Fisher Scientific). For NanoBiT assays, HEK293S cells were 535 transfected with a mixture of wt or T654A EGFR-LgBiT gene (1 $\mu \mathrm{g}$ each) and GBR2536 SmBiT $(0.2 \mu \mathrm{g})$ using Lipofectamine 3000 Reagent in $60 \mathrm{~mm}$ dish.

\section{PMA treatment and EGF stimulation}

539 DNA constructs of full-length wt and T654A EGFR were transfected and cultured with $54010 \%$ fetal bovine serum (FBS) on the day before each measurement. Cells were then 541 starved in modified Eagle's medium without FBS for 3 hours before the experiment. 542 Phorbol 12-myristate 13-acetate (PMA) was dissolved in DMSO and subsequently 543 diluted in PBS to a final concentration of $10 \mu \mathrm{M}$. For PMA pre-treatment, PMA solution 544 was added to the cell cultured medium at a final concentration of $100 \mathrm{nM}$ and incubated 545 for $30 \mathrm{~min}$ at room temperature. For EGF stimulation, EGF (PeproTech, Cranbury, NJ) 546 dissolved in PBS was added to the cell cultured medium at a final concentration of 100 $547 \mathrm{ng} / \mathrm{mL}$ (for western blotting assays and single-molecule measurements) or as a 0.001 to $100 \mathrm{nM}$ dilution series (for the NanoBiT assay).

\section{Western blotting analysis}

551 In cells stimulated with $\mathrm{EGF}$ for $0,5,30 \mathrm{~min}$ at $37^{\circ} \mathrm{C}$, threonine and tyrosine 552 phosphorylation of the wt and mutant T654A proteins was detected by western blotting 553 using rabbit anti-pT654 (ab75986; Abcam, Cambridge, UK) and rabbit anti-pY1086 554 antibody (\#4407; Cell Signaling Technology, Danvers, MA), respectively. Rabbit anti555 EGFR antibody (\#sc-03; Santa Cruz Biotechnology, Dallas, TX) was used to detect 556 protein expression. After being resolved by SDS-polyacrylamide gel electrophoresis 557 (PAGE), the electrophoresed proteins were transferred onto a polyvinylidene difluoride 558 (PVDF) membrane and incubated with each antibody (primary antibody) and then with a 559 horseradish peroxidase (HRP)-linked anti-rabbit IgG (secondary antibody; 7076, Cell 
560 Signaling Technology). Immunoreactive proteins were detected with Amersham ECL

561 Prime Western Blotting Detection Reagent (GE Healthcare) using an ImageQuant LAS

562500 device (GE Healthcare).

563

\section{Single-molecule imaging in living cells}

565 The methods for single-molecule measurement and analysis were described elsewhere

566 (Yanagawa and Sako, Methods in Mol Biol, in press; bioRxiv: doi: 567 10.1101/2020.06.08.141192). The single-molecule imaging of EGFR was performed at 568 the basal plasma membrane of the $\mathrm{CHO}-\mathrm{K} 1$ cells at $25^{\circ} \mathrm{C}$ with the same microscopic 569 methods used for the spFRET measurements. The laser wavelength was $488 \mathrm{~nm}$ (Sapphire 570 488; Coherent, Santa Clara, CA) for the excitation of the GFP. Fluorescence images were 571 acquired every $50 \mathrm{~ms}$ using AIS software. The acquired multiple TIFF files were 572 processed by ImageJ software as follows: background subtraction was performed with a 573 rolling ball radius of 25 pixels, and two-frame averaging of the images was then 574 performed. Single-molecule tracking analysis was performed with AAS software (ZIDO). 575 All subsequent analyses were performed using smDynamicsAnalyzer 576 (https://github.com/masataka-yanagawa/IgorPro8-smDynamicsAnalyzer), an Igor Pro 577 8.0-based homemade program.

\section{NanoBiT assay}

580 HEK293S cells co-transfected with the plasmids of wt or T654A EGFR-LgBiT and 581 GRB2-SmBiT. Overnight after the transfection, cells were collected in $0.5 \mathrm{mM}$ EDTAcontaining PBS, centrifuged, and suspended in $2 \mathrm{~mL}$ of HBSS containing $0.01 \%$ bovine serum albumin and $5 \mathrm{mM}$ HEPES ( $\mathrm{pH}$ 7.4) (assay buffer). The cell suspension was dispensed in a 96-well white bottom plate at a volume of $80 \mu \mathrm{L}$ per well and loaded with $20 \mu \mathrm{L}$ of $25 \mu \mathrm{M}$ Nano-Glo Vivazine Live Cell Substrates (Promega) diluted in the assay buffer. After incubation for $2 \mathrm{hrs}$ at room temperature in the assay buffer, cells were pretreated with PMA or vehicle as described above. Basal luminescence was then measured by using a microplate leader (SpectraMax L, Molecular Devices) with an interval of $60 \mathrm{sec}$ at room temperature. After $10 \mathrm{~min}, 20 \mu \mathrm{L}$ of the EGF dilution series in the assay buffer or the assay buffer (vehicle) were applied to each well using a benchtop multi-pipetter (EDR-384SR, BioTec, Tokyo, Japan) under red dim light. Then, luminescence was measured for $30 \mathrm{~min}$ with an interval of $60 \mathrm{sec}$. Each time-course of luminescence counts was normalized with the luminescence counts of the vehicle-added well. Dose-response curves were fitted with a Hill-equation to determine the maximum intensity. 


\section{Acknowledgments}

597 YS was supported by MEXT Japan with Grants-in-Aid for Scientific Research

598 (19H05647) and by JST CREST (JPMJCR1912). We thank Hiromi Sato for technical 599 assistance.

600

601 Author contributions

602 Conceptualization, R.M., Y.S.; methodology, R.M., T.S., M.Y., Y.S.; investigation, R.M., 603 H. T., T. S., M.Y.; manuscript writing, R.M., T. S., Y.S. with feedback from all other 604 coauthors; funding acquisition, Y.S.; supervision, Y.S.

605

606 Conflicts of interest

607 None.

608 


\section{References}

610 1. M. A. Olayioye, R. M. Neve, H. A. Lane, N. E. Hynes, The ErbB signaling network:

611 receptor heterodimerization in development and cancer. EMBO J.19, 3159-3167

$612 \quad$ (2000).

$6132 . \quad$ M. K. Nyati, M. A. Morgan, F. Y. Feng, T. S. Lawrence, Integration of EGFR inhibitors 614 with radiochemotherapy. Nat. Rev. Cancer 6, 876-885 (2006).

615 3. E. Kovacs, J. A. Zorn, Y. Huang, T. Barros, J. Kuriyan, A structural perspective on the 616 regulation of the epidermal growth factor receptor. Annu. Rev. Biochem. 84, 739-764 $617 \quad$ (2015)

618 4. N. J. Bessman, M. A. Lemmon, Finding the missing links in EGFR. Nat. Struct. Mol. 619 Biol. 19, 1-3 (2012).

620 5. H. Ogiso et al., Crystal structure of the complex of human epidermal growth factor and receptor extracellular domains. Cell110, 775-787 (2002).

6. X. Zhang, J. Gureasko, K. Shen, P. A. Cole, J. Kuriyan, An allosteric mechanism for activation of the kinase domain of epidermal growth factor receptor. Cell 125, 11371149 (2006).

7. M. J. Wagner, M. M. Stacey, B. A. Liu, T. Pawson, Molecular mechanisms of SH2- and PTB-domain-containing proteins in receptor tyrosine kinase signaling. Cold Spring Harb Perspect Biol 5, a008987 (2013).

8. K. M. Ferguson et al., EGF activates its receptor by removing interactions that autoinhibit ectodomain dimerization. Mol. Cell 11, 507-517 (2003).

9. N. F. Endres et al., Conformational coupling across the plasma membrane in activation of the EGF receptor. Cell 152, 543-556 (2013).

10. E. R. Wood et al., A unique structure for epidermal growth factor receptor bound to GW572016 (Lapatinib): relationships among protein conformation, inhibitor off-rate, and receptor activity in tumor cells. Cancer Res. 64, 6652-6659 (2004).

11. N. Jura et al., Mechanism for activation of the EGF receptor catalytic domain by the juxtamembrane segment. Cell 137, 1293-1307 (2009).

12. S. E. Webb et al., Single-molecule imaging and fluorescence lifetime imaging microscopy

640 13. Y. Huang et al., Molecular basis for multimerization in the activation of the epidermal show different structures for high- and low-affinity epidermal growth factor receptors in growth factor receptor. Elife 5 (2016). 
645

646

647

648

649

650

651

652

653

654

655

656

657

658

659

660

661

662

663

664

665

666

667

668

669

670

671

672

673

674

675

676

677

678

679

680

15. N. Jura, Y. Shan, X. Cao, D. E. Shaw, J. Kuriyan, Structural analysis of the catalytically inactive kinase domain of the human EGF receptor 3. Proc Natl Acad Sci U S A 106, 21608-21613 (2009).

16. S. J. Fleishman, J. Schlessinger, N. Ben-Tal, A putative molecular-activation switch in the transmembrane domain of erbB2. Proc Natl Acad Sci U S A 99, 15937-15940 (2002).

17. A. Arkhipov et al., Architecture and membrane interactions of the EGF receptor. Cell 152, 557-569 (2013).

18. M. Lelimousin, V. Limongelli, M. S. Sansom, Conformational Changes in the Epidermal Growth Factor Receptor: Role of the Transmembrane Domain Investigated by CoarseGrained MetaDynamics Free Energy Calculations. J. Am. Chem. Soc. 138, 10611-10622 (2016).

19. C. Matsushita et al., Transmembrane helix orientation influences membrane binding of the intracellular juxtamembrane domain in Neu receptor peptides. Proc Natl Acad Sci U $S A$ 110, 1646-1651 (2013).

20. R. Maeda, T. Sato, K. Okamoto, M. Yanagawa, Y. Sako, Lipid-Protein Interplay in Dimerization of Juxtamembrane Domains of Epidermal Growth Factor Receptor. Biophys. J. 114, 893-903 (2018).

21. K. B. Abd Halim, H. Koldso, M. S. P. Sansom, Interactions of the EGFR juxtamembrane domain with PIP2-containing lipid bilayers: Insights from multiscale molecular dynamics simulations. Biochim Biophys Acta 1850, 1017-1025 (2015).

22. Y. Wang et al., Regulation of EGFR nanocluster formation by ionic protein-lipid interaction. Cell Res. 24, 959-976 (2014).

23. Y. Liu et al., The involvement of lipid rafts in epidermal growth factor-induced chemotaxis of breast cancer cells. Mol. Membr. Biol. 24, 91-101 (2007).

24. T. Furuchi, R. G. Anderson, Cholesterol depletion of caveolae causes hyperactivation of extracellular signal-related kinase (ERK). J. Biol. Chem. 273, 21099-21104 (1998).

25. T. Ringerike, F. D. Blystad, F. O. Levy, I. H. Madshus, E. Stang, Cholesterol is important in control of EGF receptor kinase activity but EGF receptors are not concentrated in caveolae. J. Cell Sci. 115, 1331-1340 (2002).

26. K. A. Lund et al., Phosphorylation of the Epidermal Growth-Factor Receptor at Threonine 654 Inhibits Ligand-Induced Internalization and down-Regulation. J. Biol. Chem. 265, 20517-20523 (1990).

27. M. Hiroshima, Y. Saeki, M. Okada-Hatakeyama, Y. Sako, Dynamically varying interactions between heregulin and ErbB proteins detected by single-molecule analysis in living cells. Proc Natl Acad Sci U S A 109, 13984-13989 (2012). 
681 28. M. Yasui, M. Hiroshima, J. Kozuka, Y. Sako, M. Ueda, Automated single-molecule imaging in living cells. Nat Commun 9, 3061 (2018).

29. A. S. Dixon et al., NanoLuc Complementation Reporter Optimized for Accurate Measurement of Protein Interactions in Cells. ACS Chem Biol 11, 400-408 (2016).

30. T. Sato, P. Pallavi, U. Golebiewska, S. McLaughlin, S. O. Smith, Structure of the membrane reconstituted transmembrane-juxtamembrane peptide EGFR(622-660) and its interaction with Ca2+/calmodulin. Biochemistry 45, 12704-12714 (2006).

31. D. H. Jones, K. R. Barber, C. W. Grant, The EGF receptor transmembrane domain: 2H NMR study of peptide phosphorylation effects in a bilayer environment. Biochemistry 37, 7504-7508 (1998).

32. K. Matsuzaki, Why and how are peptide-lipid interactions utilized for self-defense? Magainins and tachyplesins as archetypes. Biochim Biophys Acta 1462, 1-10 (1999).

33. M. Hiroshima et al., Membrane cholesterol interferes with tyrosine phosphorylation but facilitates the clustering and signal transduction of EGFR. bioRxiv 10.1101/2021.08.28.457965 (2021).

34. M. Red Brewer et al., The juxtamembrane region of the EGF receptor functions as an activation domain. Mol. Cell 34, 641-651 (2009).

35. X. Li, Y. Huang, J. Jiang, S. J. Frank, ERK-dependent threonine phosphorylation of EGF receptor modulates receptor downregulation and signaling. Cell. Signal. 20, 2145-2155 (2008).

36. H. J. Zhu, J. Iaria, S. Orchard, F. Walker, A. W. Burgess, Epidermal growth factor receptor: association of extracellular domain negatively regulates intracellular kinase activation in the absence of ligand. Growth Factors 21, 15-30 (2003). regulate clathrin-mediated endocytosis of the epidermal growth factor receptor. J. Cell Biol. 189, 871-883 (2010). as a Novel Negative Feedback Mechanism of EGFR Signaling. PLoS One 10, e0139971 (2015).

39. K. Oda, Y. Matsuoka, A. Funahashi, H. Kitano, A comprehensive pathway map of epidermal growth factor receptor signaling. Mol. Syst. Biol. 1, 20050010 (2005). domain of the EGF receptor inhibits calmodulin binding. Biochem. Biophys. Res.

715 41. K. Kojima, Y. Imamoto, R. Maeda, T. Yamashita, Y. Shichida, Rod visual pigment optimizes active state to achieve efficient $\mathrm{G}$ protein activation as compared with cone 
visual pigments. J. Biol. Chem. 289, 5061-5073 (2014).

718 42. M. Pirchi et al., Single-molecule fluorescence spectroscopy maps the folding landscape of a large protein. Nat Commun 2, 493 (2011).

43. R. Lamichhane et al., Single-molecule view of basal activity and activation mechanisms of the G protein-coupled receptor $\beta_{2}$ AR. Proc. Natl. Acad. Sci. U. S. A. 112, 1425414259 (2015).

44. R. Maeda et al., Single-molecule observation of the ligand-induced population shift of rhodopsin, a G-protein-coupled receptor. Biophys. J. 106, 915-924 (2014).

45. K. Okamoto, Y. Sako, Variational Bayes Analysis of a Photon-Based Hidden Markov 


\section{Figures}

729 Figure 1. Construction of nanodiscs containing fluorescent TM-JM peptides of EGFR.

730 (a) Amino acid sequence of the EGFR TM and JM-A domains. EX, extracellular domain;

731 TK, tyrosine kinase domain. (b, c) Schematic images of nanodiscs containing dimeric

732 TM-JM peptides fluorescently labeled at the $\mathrm{N}$ - (b) and C-terminus (c), respectively. (d)

733 Lipid compositions used in the preparation of each nanodisc sample. The fractional ratios

734 of PS and cholesterol mimic those in the mammalian plasma membranes. (e) Size-

735 exclusion chromatography used for the purification of nanodiscs containing cholesterol

736 (red) or not (black). The charge ratios of PC/PS/cholesterol are described in the upper

737 table. The fraction having a peak absorbance of around 12 elution volumes $(\mathrm{mL})$ was

738 collected and used for the subsequent experiments. (f) A thin-layer chromatography of

739 the nanodisc fraction containing cholesterol (fraction 12 in (e)). (g) A negative stain

740 electron micrograph of fraction 12 in (e). Size distribution of the nanodiscs calculated

741 from the images was fitted with a Gaussian function, with a mean diameter of $11 \pm 2 \mathrm{~nm}$.

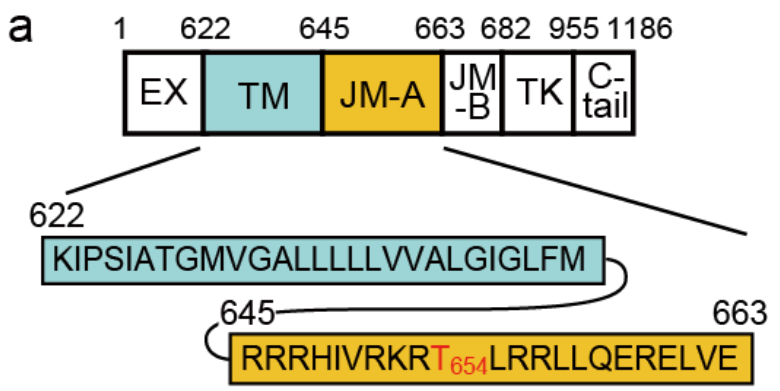

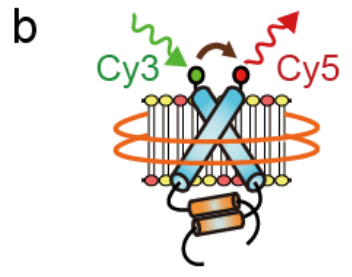

TM-TM
C

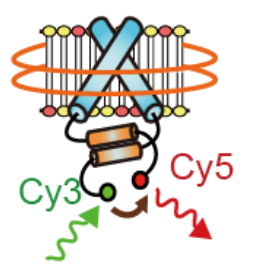

JM-JM

d

\begin{tabular}{ccccc} 
d & \multicolumn{2}{c}{ cholesterol (-) } & \multicolumn{2}{c}{ cholesterol $(+)$} \\
\cline { 2 - 5 } & PC only PC + PS PC only PC + PS \\
\hline POPC & 100.0 & 75.0 & 50.0 & 37.5 \\
\hline POPS & 0.0 & 25.0 & 0.0 & 12.5 \\
\hline cholesterol & 0.0 & 0.0 & 50.0 & $50.0 \%$
\end{tabular}
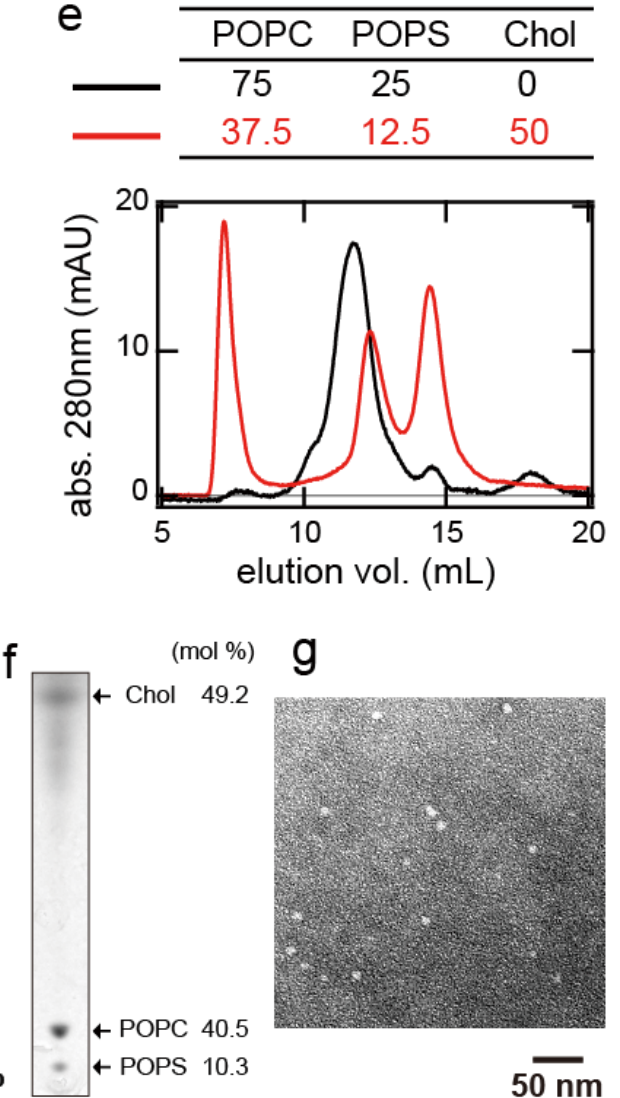

g

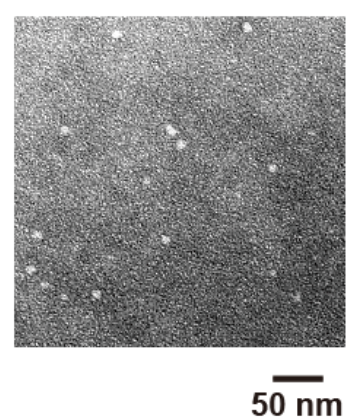


744 Figure 2. Single-pair FRET measurement of the EGFR TM-JM dimers in nanodiscs. (a)

745 Fluorescence micrograph of nanodiscs illuminated with a green laser. Cy3 (green) and

746 Cy5 (red) emissions were superimposed. The Cy5 emission was caused by FRET from

747 Cy3. (b) Representative fluorescence trajectories of Cy3 (green) and Cy5 (red). Black

748 allows indicate photobleaching points of Cy5. (c) FRET efficiency trajectories of the

749 fluorescence trajectories in (b). The FRET efficiency, $E_{\mathrm{FRET}}$, was calculated as described

750 in the Materials and Methods section. Typical fluorescence and FRET trajectories

751 between peptides labeled at the C-terminus are shown. Transitions to low FRET

752 efficiency states suggested that dissociation of the JM dimer occurred occasionally. The

753 Förster radius $R_{0}$ between $\mathrm{Cy} 3$ and $\mathrm{Cy} 5$ is $5.6 \mathrm{~nm}$.

a
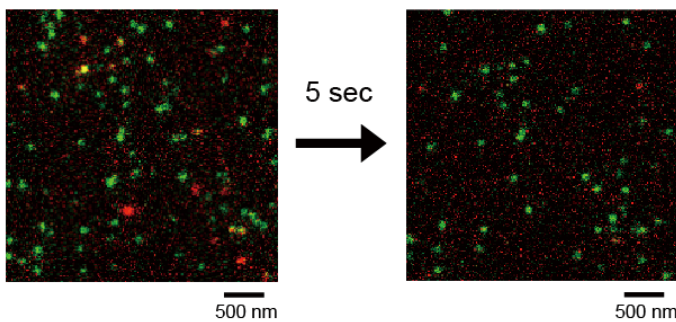

b

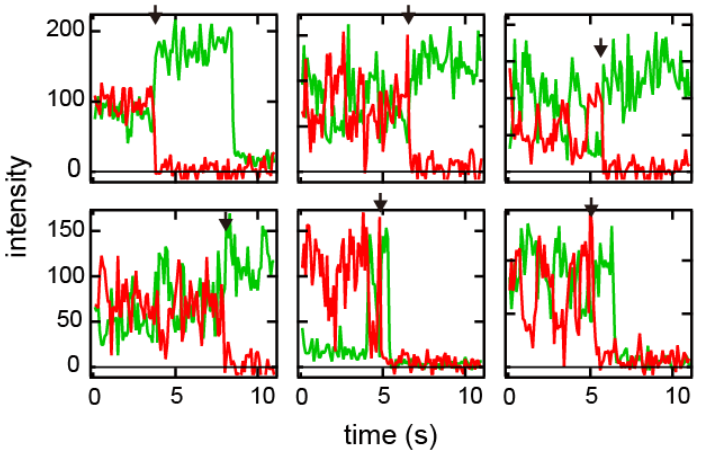

C

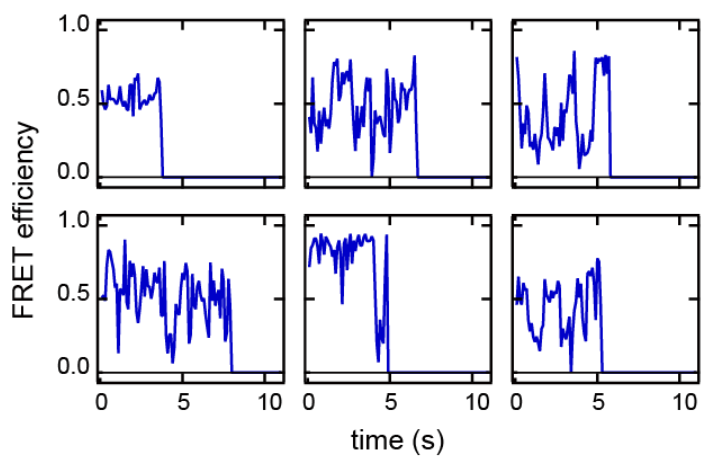


757 Figure 3. FRET efficiency $\left(E_{\mathrm{FRET}}\right)$ distributions in nanodiscs containing a single Cy3/Cy5-pair of N-terminal labeled peptides. Nanodiscs contained non-phosphorylated (a-d) and Thr654 phosphorylated (e-h) peptides at the indicated lipid conditions. Positions of the mode and its 5-95\% percentile section are indicated by solid and dashed lines, respectively. See Table I for these values. In (b-h), the distribution and the 95\% percentile section shown in (a) are superimposed (red) for comparison.

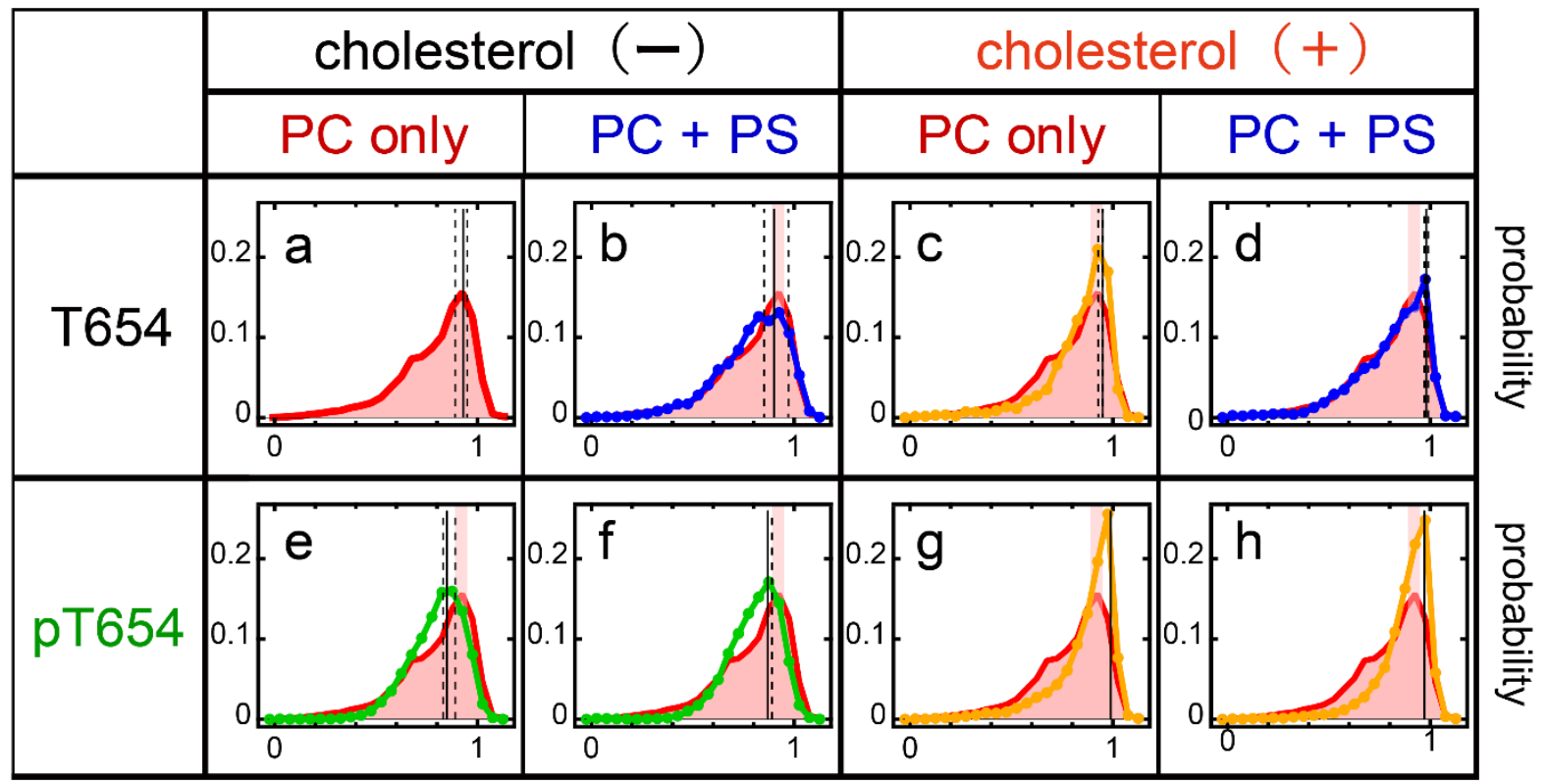

FRET efficiency

FRET efficiency 
bioRxiv preprint doi: https://doi org/10.1101/2021 05.14.444132. this version posted September 6, 2021. The copyright holder for this preprint (which was not certified by peer review) is the author/funder, who has granted bioRxiv a license to display the preprint in perpetuity. It is made available under aCC-BY-NC-ND 4.0 International license.

767 Figure 4. $E_{\mathrm{FRET}}$ distributions in nanodiscs containing a single Cy3/Cy5-pair of C-terminal labeled peptides. Nanodiscs contained non-phosphorylated (a-d) and Thr654 phosphorylated $(\mathbf{e}-\mathbf{h})$ peptides in the indicated lipid conditions. Positions of the mode and its $5-95 \%$ percentile section are indicated by solid and dashed lines, respectively. See Table I for these values. In (b-h), the distribution and the 95\% percentile section shown in (a) are superimposed (red) for comparison.

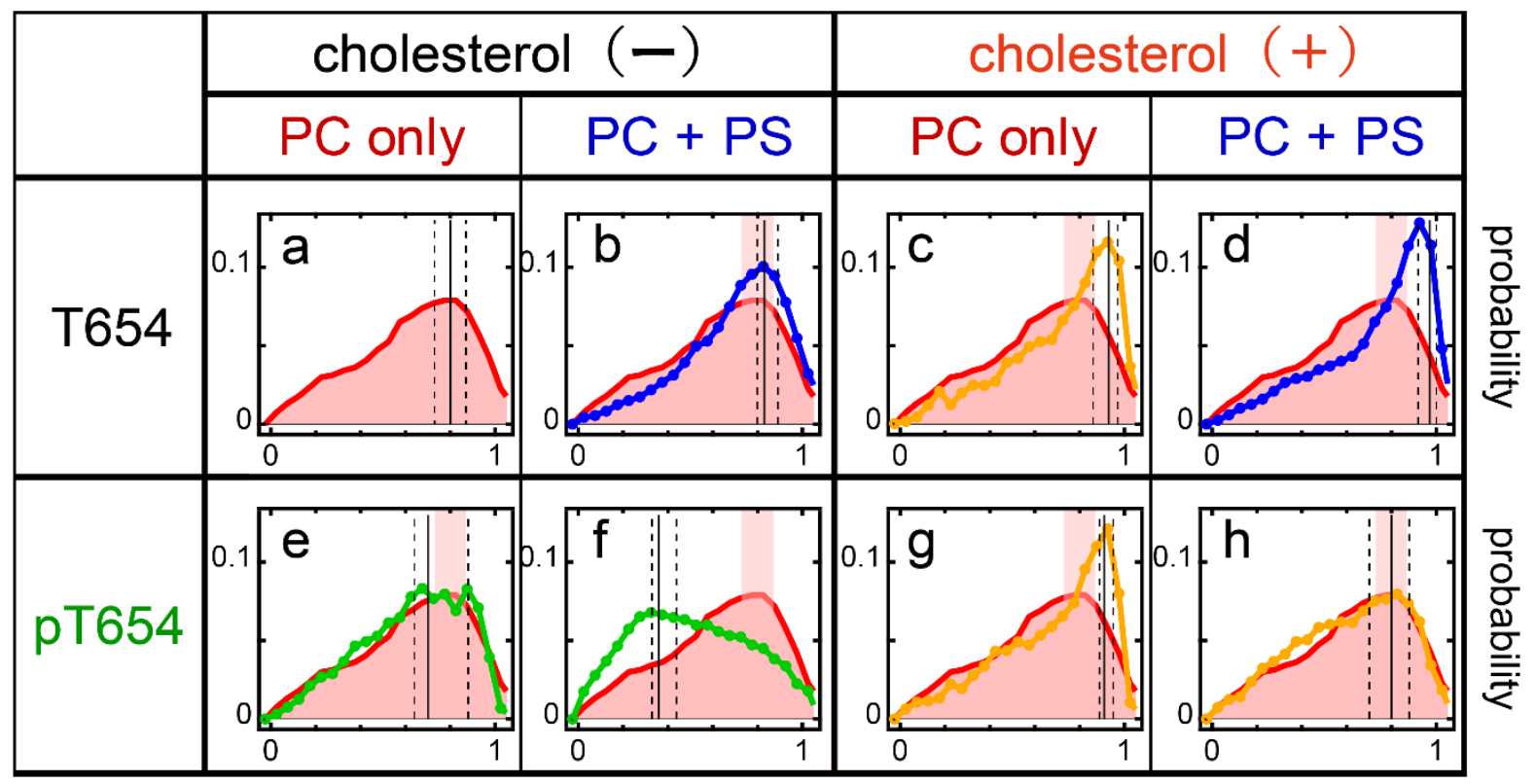

FRET efficiency

FRET efficiency 
777 Figure 5. Higher-order oligomerization of EGFR TM-JM peptides in the nanodiscs.

778 Histograms are shown of the total fluorescence intensity of the peptides with Cy3-labeling 779 at the C-terminus in single nanodiscs containing cholesterol (blue) or not (red). Discs 780 containing no Cy5 peptide were chosen for measurement to avoid the possible effects of 781 FRET. Peptides with a non-phosphorylated (a, c) or phosphorylated (b, d) Thr654 were reconstituted into nanodiscs in PC $(\mathbf{a}, \mathbf{b})$ or PC/PS $(\mathbf{c}, \mathbf{d})$ membranes.

a

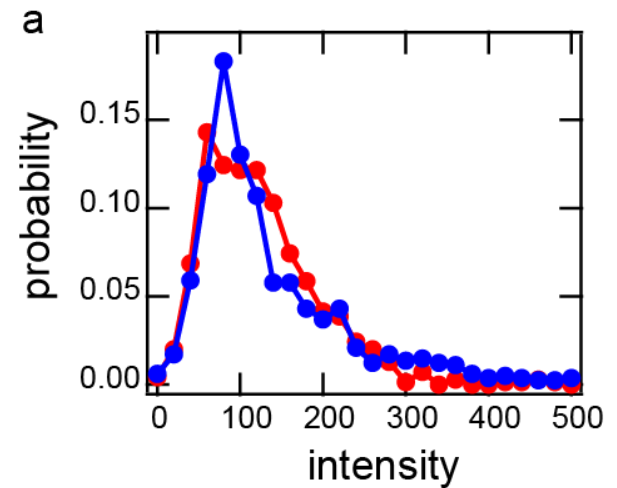

C

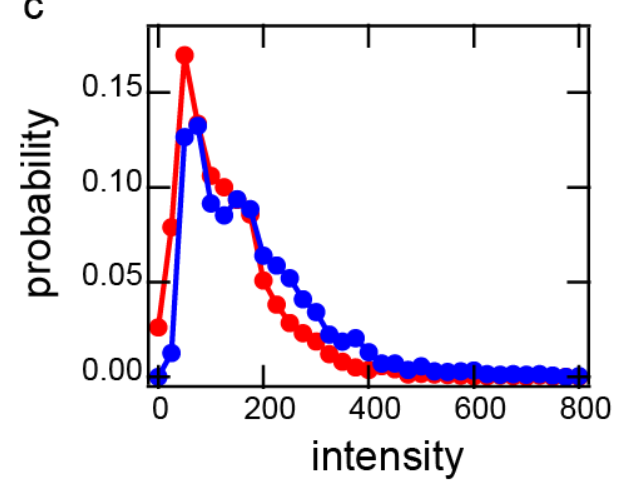

b

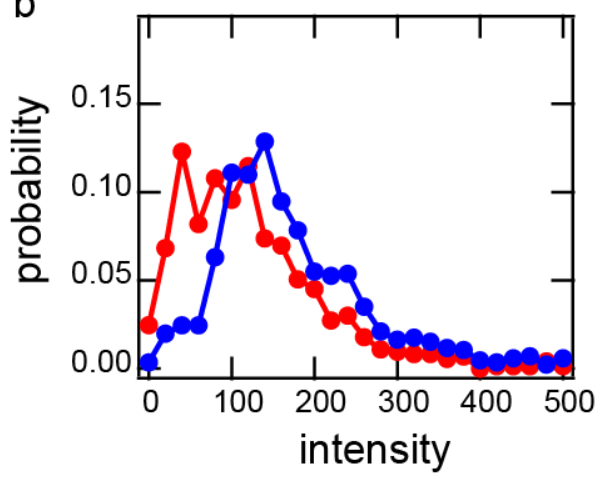

d

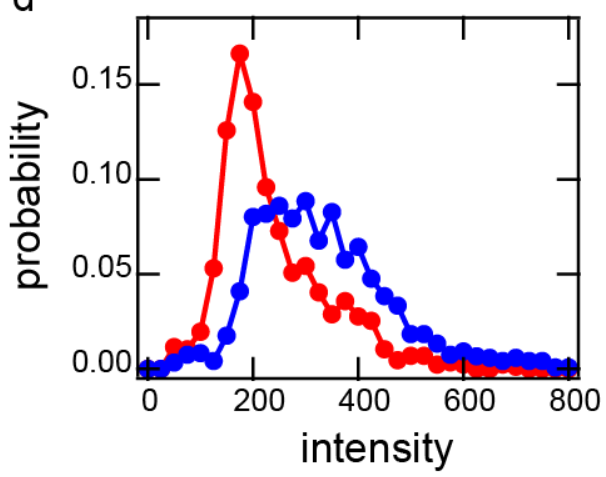


785 Figure 6. Assembly of EGFR TM regions. (a) Representative fluorescence trajectories of 786 Cy3 (green) and Cy5 (red) in nanodiscs containing two Cy3-labeled and one Cy5-labeled 787 peptide. Fluorescence intensities and/or two-step photobleaching dynamics after Cy5 788 photobleaching indicated that these nanodiscs contained two Cy3 peptides. (b-i) 789 Fluorescence intensity histograms of N-terminal-labeled $\mathrm{Cy} 3$ peptides before (blue) and 790 after (red) Cy5 photobleaching. Nanodiscs contained non-phosphorylated (b-e) and 791 Thr654 phosphorylated (f-i) peptides at the indicated lipid conditions. (j) Schematic 792 structures indicating proximity between three TM domains before (left) and after (right) 793 Cy5 photobleaching. Note that acceptance of the excitation energy from Cy3 was not 794 saturated for Cy5 under our experimental conditions, even in the presence of two Cy3 795 molecules.

\section{a}
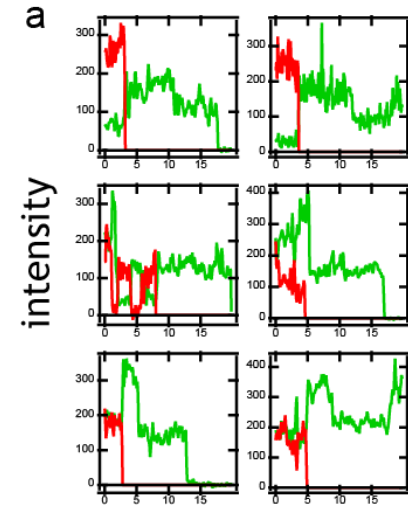

time $(\mathrm{sec})$ j trimer

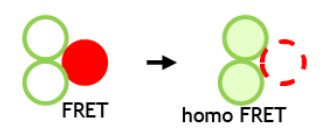

dimer + monomer

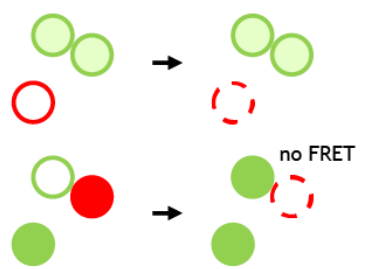

pre-bleach after bleach

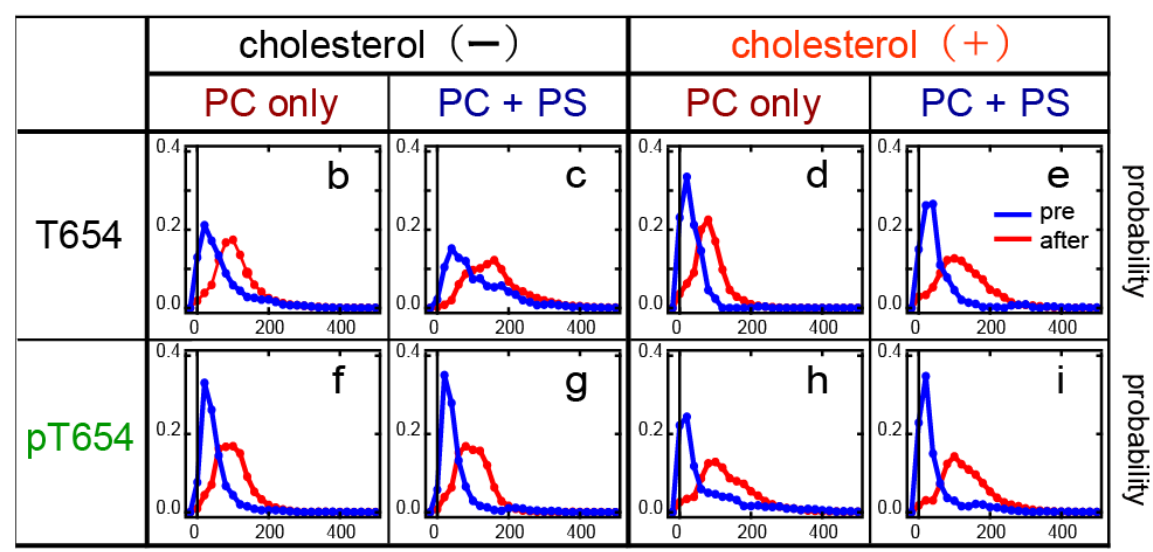

intensity

intensity 
798 Figure 7. Assembly of EGFR JM regions. (a) Representative fluorescence trajectories of 799 Cy3 (green) and Cy5 (red) in nanodiscs containing two Cy3-labeled and one Cy5-labeled

800 peptide at the C-terminus. (b-i) Fluorescence intensity histograms of C-terminal-labeled 801 Cy3 peptides from nanodiscs containing two Cy3 and one Cy5 peptide before (blue) and 802 after (red) Cy5 photobleaching. Nanodiscs contained non-phosphorylated (b-e) and 803 Thr654 phosphorylated (f-i) peptides at the indicated lipid conditions. (j) Schematic 804 structures indicating proximity between three JM domains before (left and middle) and 805 after (right) Cy5 photobleaching.

a
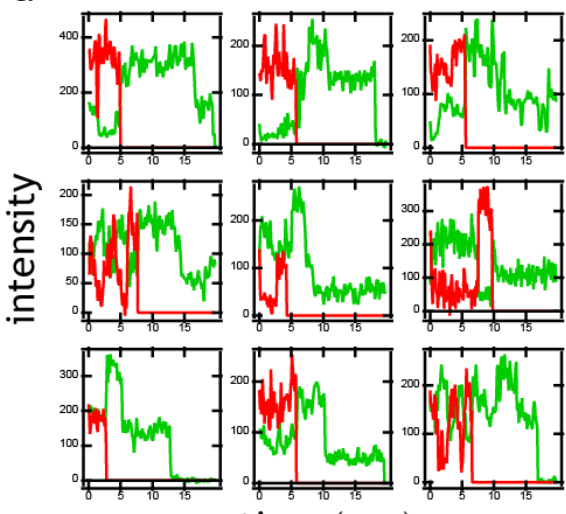

time (sec)

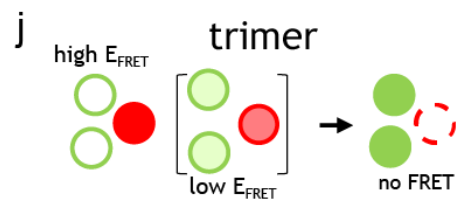

dimer + monomer

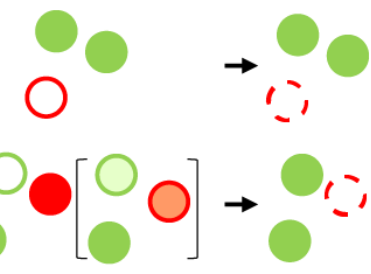

pre-bleach after bleach

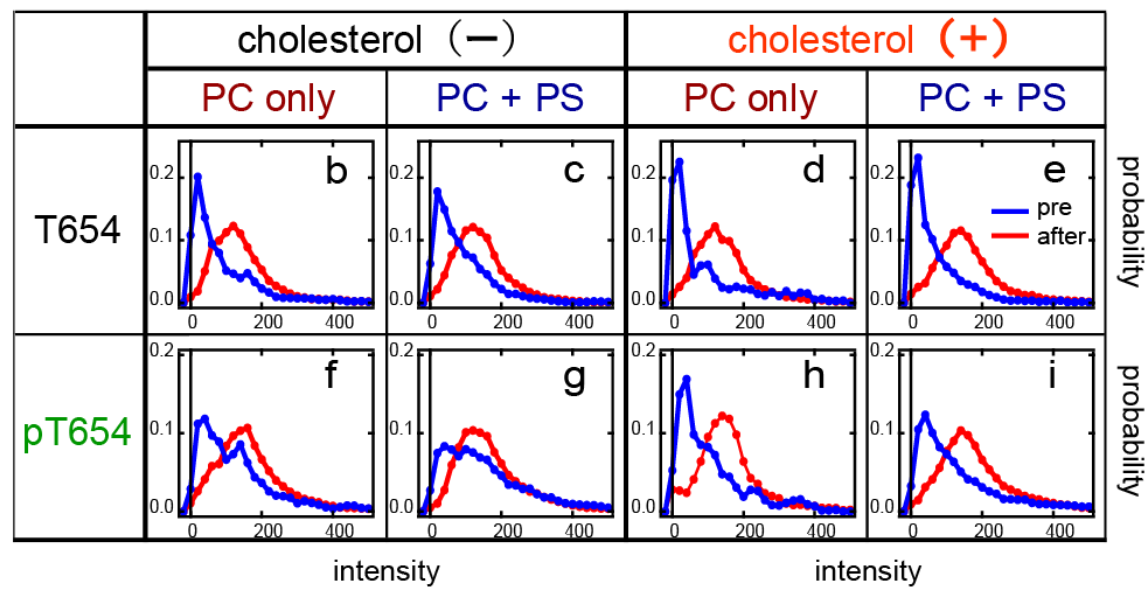


bioRxiv preprint doi: https://doi org/10.1101/2021.05.14.444132. this version posted September 6, 2021. The copyright holder for this preprint (which was not certified by peer review) is the author/funder, who has granted bioRxiv a license to display the preprint in perpetuity. It is made available under aCC-BY-NC-ND 4.0 International license.

808 Figure 8. Possible configurations of EGFR TM-JM dimers and trimers regulated by 809 membrane lipids and Thr654 phosphorylation.

810

non-phosphorylated

a

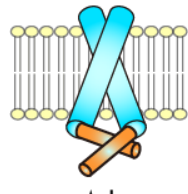

$$
\mathrm{PC} \uparrow \mathrm{PS}
$$
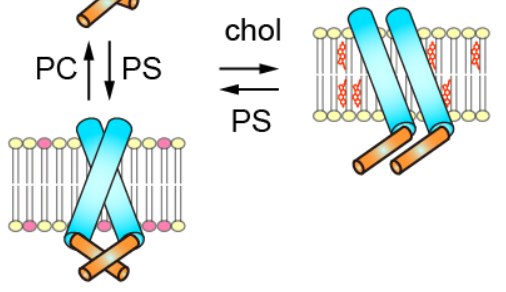

C
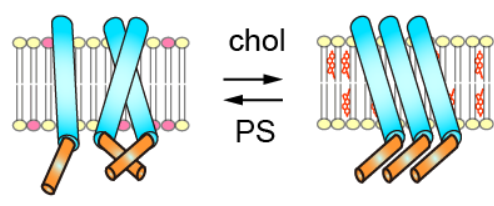

b

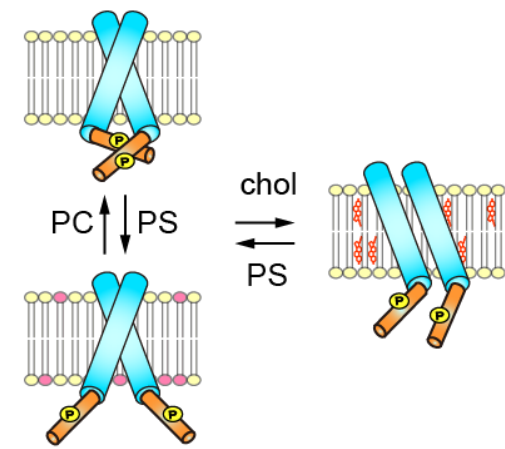

d

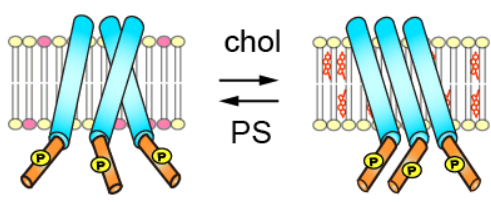


812 Figure 9. Thr and Tyr phosphorylation of EGFR. (a) Thr654 phosphorylation after EGF 813 stimulation and PMA pretreatment. (b, c) Timecourses of Y1068 phosphorylation for the 814 wt and T654A mutant of EGFR during EGF stimulation. Typical western blotting results 815 are indicated (b, top) and the average of four independent experiments are shown with 816 SE (c). Phosphorylation levels were normalized to the expression levels of the whole 817 EGFR molecule (b, bottom).

818

a

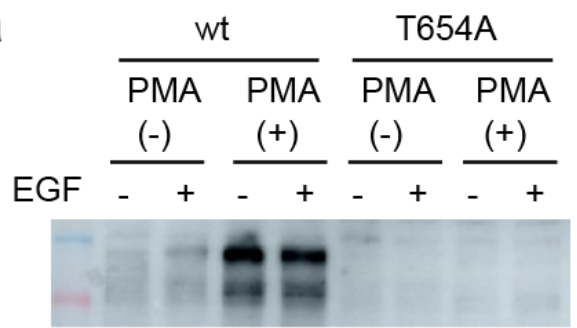

b

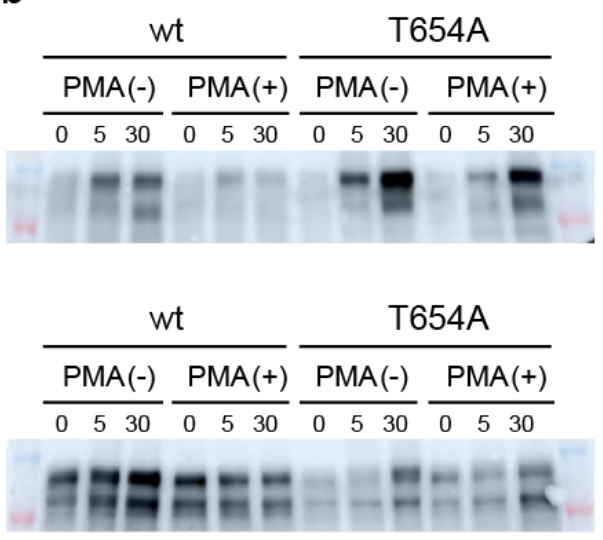

C

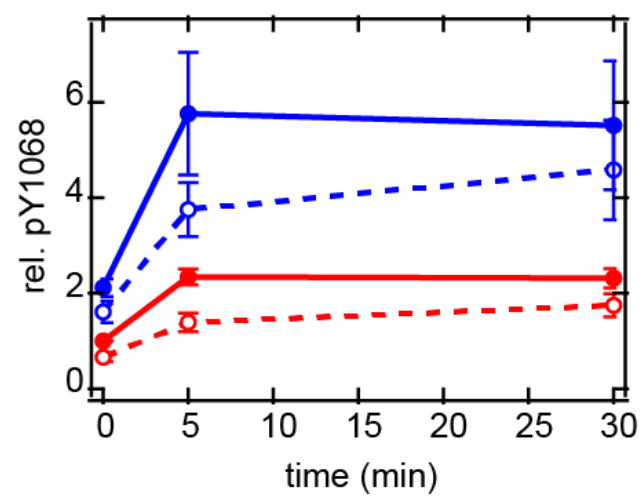

$$
\begin{array}{ll}
\text { wt, PMA (-) } & (n=4) \\
\text { wt, PMA (+) } & (n=4) \\
\text { T654A, PMA (-) } & (n=4) \\
\text { T654A, PMA (+) } & (n=4)
\end{array}
$$


820 Figure 10. Oligomerization and lateral movements of EGFR on the living cell surface.

821 (a) Single molecule imaging of wt EGFR-GFP on the surface of living CHO-K1 cells 822 with (right) and without (left) PMA pretreatment. Cells were stimulated with (lower) and 823 without (upper) EGF. Bar, $5 \mu \mathrm{m}$. Quantized transitions in the fluorescence intensities 824 (bottom) indicate a single-molecule resolution of the imaging. (b) Oligomer size 825 distributions of wt (left) and T654A mutant (right) EGFR in cells. The oligomer size ratio 826 was measured before and $10 \mathrm{~min}$ after EGF stimulation. (c) Mean square displacement 827 (MSD) of wt (left) and T654A (right) EGFR spots as a function of the time interval, 828 indicating lateral mobility. The MSD was calculated before and 10 min after EGF 829 stimulation. In (b, c), cells were pretreated with (blue, green) or without (black, red) PMA 830 and stimulated (red, green) or not (black, blue) with EGF. (d) Diagram of the 831 oligomerization and immobilization states of wt (left) and T654A mutant (right) EGFR 832 suggested from single-molecule measurements. Arrows indicate the state transitions after 833 PMA treatment and EGF stimulation.

a
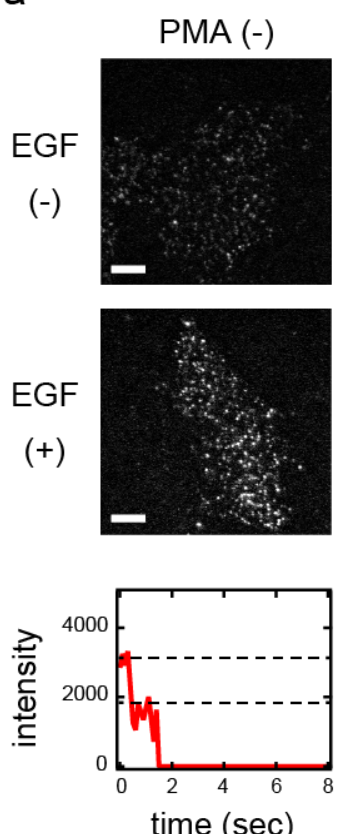

time (sec)
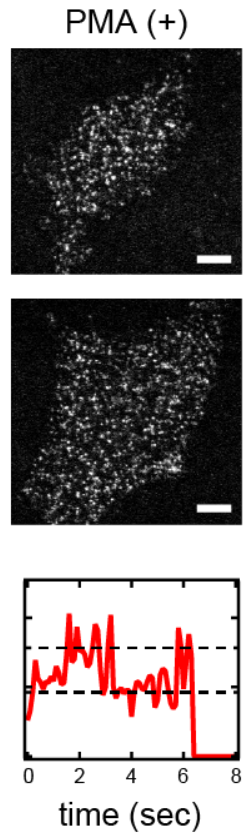

EGF (-), EGF (+) PMA (+) , PMA (+) / EGF (+)
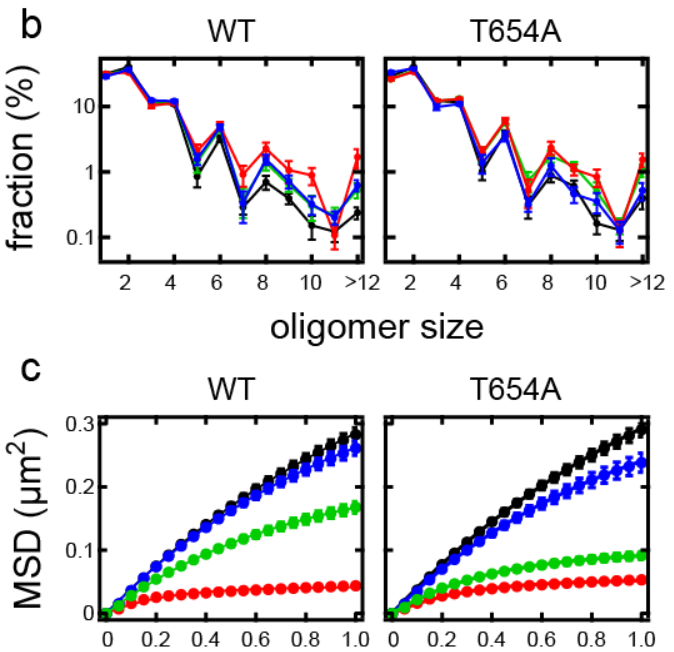

$\Delta \mathrm{t}(\mathrm{s})$

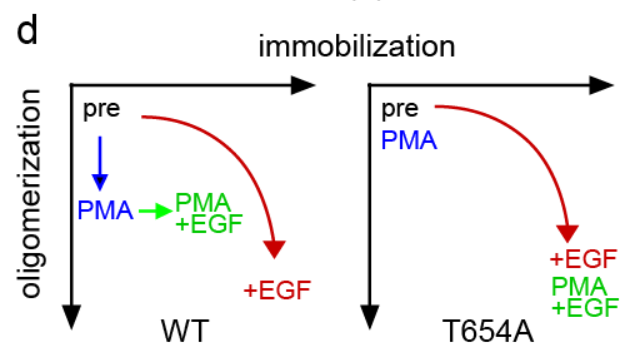


838 Figure 11. NanoBiT assay for the EGFR/GRB2 interaction in living cells. (a) Schematic 839 illustration of the NanoBiT assay of EGFR/GRB2 interactions. (b) Typical time courses 840 of chemiluminescence signals generated from the complex formation of large BiT 841 (LgBiT)-fused EGFR and small BiT (SmBiT)-fused GRB2 after EGF application at time 8420 . The final concentration of EGF in the culture medium was varied from 0.0001 to 100 843 nM. Measurements were done in cells with (+) or without (-) PMA pretreatment. (c) Dose 844 dependency of the chemiluminescence intensities at $30 \mathrm{~min}$ after EGF stimulation. The 845 average values from four independent experiments are shown with SE. Lines indicate 846 fitting with a Hill-equation function. ${ }^{*} \mathrm{p}<0.05$ determined by $t$-test against the signal in 847 wt cells without PMA. (d) A schematic model of the activation and signal transduction 848 process for EGFR dimers and oligomers. In the oligomers of EGFR, affinity with $\mathrm{GRB}_{2}$ 849 is increased.
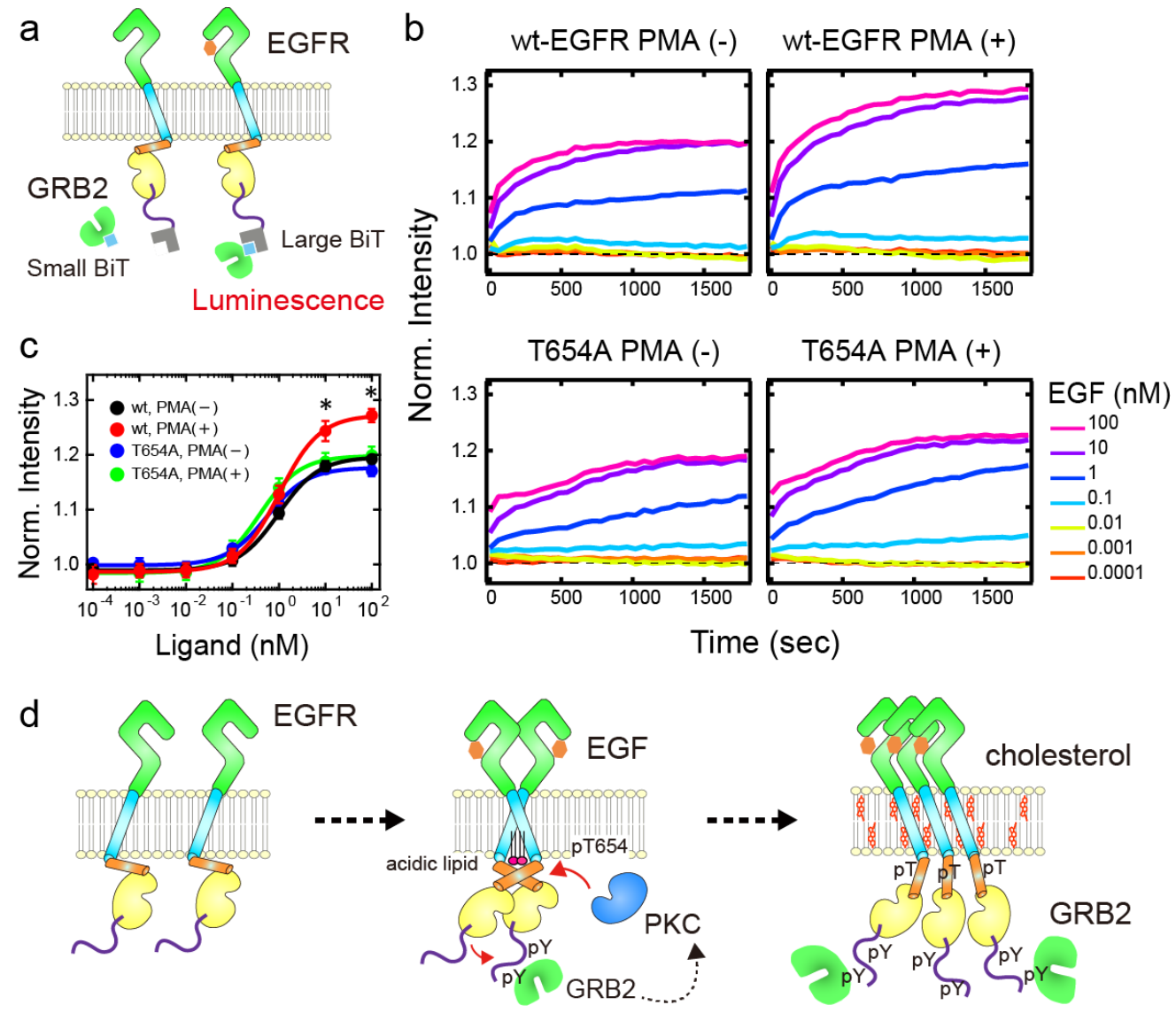
852 Table I. Peak values in the $E_{F R E T}$ distributions between the TM-JM peptides

853

\begin{tabular}{|c|c|c|c|c|c|}
\hline \multirow{3}{*}{ lipid composition } & \multirow{3}{*}{ pT654 } & \multicolumn{4}{|c|}{ labelling } \\
\hline & & \multicolumn{2}{|c|}{$\mathrm{N}$-term } & \multicolumn{2}{|c|}{ C-term } \\
\hline & & mean & $95 \%$ & mean & $95 \%$ \\
\hline PC & - & 0.93 & $(0.89-0.95)$ & 0.81 & $(0.73-0.87)$ \\
\hline $\mathrm{PC} / \mathrm{PS}$ & - & 0.90 & $(0.85-0.97)$ & 0.83 & $(0.79-0.89)$ \\
\hline PC/chol & - & 0.95 & $(0.93-0.95)$ & 0.93 & $(0.85-0.97)$ \\
\hline PC/PS/chol & - & 0.98 & $(0.97-0.99)$ & 0.94 & $(0.91-0.97)$ \\
\hline PC & + & 0.85 & $(0.83-0.89)$ & 0.68 & $(0.63-0.89)$ \\
\hline $\mathrm{PC} / \mathrm{PS}$ & + & 0.87 & $(0.87-0.89)$ & 0.36 & $(0.33-0.43)$ \\
\hline PC/chol & + & 0.99 & $(0.99)$ & 0.90 & $(0.89-0.95)$ \\
\hline PC/PS/chol & + & 0.97 & $(0.97)$ & 0.82 & $(0.81-0.89)$ \\
\hline
\end{tabular}

855

856 Mean and their 95\% percentile section of the peak values in Figs 3 and 4 were estimated 857 by bootstrap method with 300-times of resampling. An increase of the resampling times 858 to 1000 did not change the result. 